\title{
The influence of adsorption layer thickness and pore geometry on gas production from tight compressible shales
}

\author{
Erfan Abolghasemi ${ }^{1,2}$, Pål Østebø Andersen ${ }^{3,4 \oplus *}$ \\ ${ }^{1}$ Department of Energy and Petroleum Engineering, University of Stavanger, 4021 Norway \\ ${ }^{2}$ Drilling and Well Department, Equinor, Bergen, 5254 Norway \\ ${ }^{3}$ Department of Energy Resources, University of Stavanger, 4021 Norway \\ ${ }^{4}$ The National IOR Centre of Norway, University of Stavanger, 4021 Norway
}

Keywords:

Adsorption model

adsorbed layer thickness

compressible shale

shale gas production

Cited as:

Abolghasemi, E., Andersen, P. Ø. The influence of adsorption layer thickness and pore geometry on gas production from tight compressible shales. Advances in Geo-Energy Research, 2021, 6(1): 4-22. https://doi.org/10.46690/ager.2022.01.02

\begin{abstract}
:
In tight shales, gas is stored in both free and adsorbed form. A one-dimensional model is derived for shale gas production by pressure depletion where the adsorbed layer thickness is of similar magnitude as the pore radius and can affect flow performance. The adsorbed layer thickness is a function of pressure. Different pore geometries are assumed varying continuously between spherical pores to more fracture shaped pores. The shale is assumed compressible and its porosity and pore radius reduce with pressure depletion. The effective pore radius (pore radius minus adsorption layer thickness) controls intrinsic and apparent permeability. The impact of the adsorption layer, compressibility and geometry are investigated. A given adsorbed layer thickness fills more of the pores when they are more spherical and concentrates more of the volumetric flow to the effective pore boundaries giving lower permeability for a given effective radius. Increasing the adsorbed thickness increases the adsorbed fraction initial gas in place. A high volume fraction adsorbed gas reduces apparent permeability and delays production. Pressure depletion causes both pore radius and adsorbed layer to be reduced. The change in adsorbed layer thickness is low at high pressure and greater at low pressure, while pore radius changes more linearly and more with higher compressibility. The free gas saturation and slip increases with pressure depletion for low compressible cases, but if matrix compression dominates there can be a net reduced permeability. Recovery was linear with the square root of time for all cases. Adsorbed gas is less effectively produced by pressure depletion than free gas and higher adsorbed content by more spherical pores or higher layer thickness reduces end recovery. Higher compressibility reduces permeability and delays recovery but increases end recovery.
\end{abstract}

\section{Introduction}

Shale gas reservoirs are organic-rich shale formations which act as both reservoir and source rock. These formations are characterized by ultra-low permeability and can contain a significant quantity of adsorbed gas in addition to free gas. The amount of adsorbed gas can vary between $20-85 \%$ of the total gas-in-place volumes (Curtis, 2002; Montgomery et al., 2005; Wu et al., 2014; Gou and Xu, 2019). The adsorbed gas is densely packed on the surface of the shale matrix and in the organic matter known as kerogen while the free gas mainly resides in nanopores and naturally occurring fractures (Yu and Sepehrnoori, 2014; Chen et al., 2019; Sheng et al., 2020). The adsorption capacity of a shale depends on factors such as total organic content, clay content, the specific surface area of nanopores and pore-size distribution (Strapoc et al., 2010; Wang et al., 2017; Klewiah et al., 2020). $\mathrm{CO}_{2}$ can adsorb preferentially over $\mathrm{CH}_{4}$ and with greater capacity, hence $\mathrm{CO}_{2}$ injection in shales can be a means to sequester $\mathrm{CO}_{2}$, mitigate climate changes and enhance shale gas production (Berawala and Andersen, 2020a; Klewiah et al., 2020). Gas adsorption

\section{Yandy
Scientific}

Press
${ }^{*}$ Corresponding author.

E-mail address: erfan18.no@gmail.com (E. Abolghasemi); pal.andersen@uis.no (P. Ø. Andersen).

Received November 11, 2021; revised November 29, 2021; accepted November 30, 2021; available online December 3, 2021. 
in shales is commonly modeled using a Langmuir isotherm (Langmuir, 1916) with the characteristic feature that substantial desorption does not occur until a significant pressure drop is obtained. Yu and Sepehrnoori (2014) history matched Marcellus and Barnett shales and found that desorption for this reason was most significant during late time production.

Technological advancements in horizontal drilling with hydraulic fracturing has made the extraction of gas from shale reservoirs economically viable. The overall recovery, which is still very low, $10-30 \%$ of the gas-in-place, however, depends on effective stimulation (Wu et al., 2014). Strong capillary or osmotic forces in shale can draw fracturing water into the matrix where it can be trapped and block gas flow (Andersen, 2021b). Typical gas production rates decline steeply after an initial peak, which affects the economics of many shale gas projects (Valko and Lee, 2010; Patzek et al., 2014). The tight nature of shales means that mainly the stimulated reservoir volume around a single well is produced effectively. Commonly, shale gas production can be studied by means of the behavior around representative individual hydraulic fractures (Berawala et al., 2019; Berawala and Andersen, 2020b).

In shales, gas transport takes place on spatial scales covering several orders of magnitude. The transport processes can effectively be divided into matrix and fractures. The flow in the matrix, which occurs on nanometer scale, is characterized by slip flow, transition flow or free molecular flow regimes based on the Knudsen number. The mean free path of gas molecules in the matrix is usually of the same order of magnitude or larger than the size of pore throat. This may cause acceleration of gas molecules leading to an increase in the apparent permeability (Tang et al., 2017). The adsorbed gas is mostly produced in the latter stages of production when a considerable amount of free gas has been depleted (Mengal and Wattenbarger, 2011). In addition to matrix flow, fracture flow is critically important in unconventional reservoirs (Zhao and $\mathrm{Du}, 2019$ ). A challenge is to open and maintain the fractures for effective gas transport.

Significant efforts have been made to effectively model the shale gas production system. A rarefaction coefficient was introduced by Beskok and Karniadakis (1999) to account for the reduced intermolecular collisions in the transition and freemolecular regimes. Javadpour (2009) introduced an apparent permeability term that includes the complexity of flow in nanopores, for application in the Darcy equation so it easily can be implemented in reservoir simulations. Civan (2010) introduced a methodology to provide accurate and meaningful correlations of data including intrinsic permeability, porosity, tortuosity effect on apparent gas permeability, rarefaction coefficient, and Klinkenberg gas slippage factor. Different modelling approaches such as molecular dynamics (Bird and Brady, 1994), direct Monte Carlo simulation (Karniadakis et al., 2006), Burnett equation (Karniadakis et al., 2006) and Lattice-Boltzmann (Hornyak et al., 2008) methods have also been implemented to model gas flow in nanopores. Characteristics like pore pressure, reservoir temperature, surface roughness, and phase change have also been studied for shale (Higashi et al., 1963; Zarragoicoechea and Kuz, 2004; Chen et al., 2008). Clarkson et al. (2012) introduced a dynamic- slippage model to study production dynamics. Knudsen diffusion was extended into the slippage factor by Civan et al. (2011) to explain gas transport. Pore transport of free and adsorbed gas was modeled by Sheng et al. (2020) and generalized to account for pore size distribution.

In addition to gas storage, adsorption can also affect gas flow relations. Xiong et al. (2012) assumed the adsorbed layer to have a thickness of one molecular diameter and calculated that a $2 \mathrm{~nm}$ pore could lose half its porosity and $75 \%$ of its permeability by adsorption. $\mathrm{Li}$ et al. (2016) modeled shale gas flow at pore scale with the Lattice-Boltzmann approach incorporating pressure dependent layer thickness. They found that permeability was reduced when the adsorbed layer filled more of the pores. Cao et al. (2016) included adsorption as a volumetric strain in a poroelastic model for shale gas. Pang et al. (2017) measured adsorption and compressibility in shale. Based on volumetric calculations the experimental effective porosity reduced when a nonadsorbing gas was present during pressure depletion, but when an adsorbing gas was present the effective porosity increased due to desorption. Similar findings were made by Memon et al. (2020). Jiang and Yang (2018) coupled a geomechanical and fluid flow model. They observed higher gas rate when the adsorbed layer was not included. Sheng and Javadpour (2018) accounted for compressibility and adsorbed volume in the organic pores and presented apparent porosity and apparent permeability formulations for use in a dual-continua reservoir model.

This paper presents a one-dimensional (1D) dynamic model for shale gas production from the matrix with $n$ spherical pore geometry. Adsorbed gas occupies portions of the volume as a finite layer and has implications on flow, storage and production dynamics. The shale is assumed compressible and its porosity and pore radius reduce with pressure depletion. Consistent relations for porosity and permeability capturing these effects are presented. The gas transport equation incorporates pore geometry as a free parameter $n$, apparent permeability, compressibility, adsorption layer thickness and free gas saturation. The system is solved numerically using operator splitting and experimental data from the literature are used as input with significant adsorption and compressibility effects. The proposed model addresses some questions of practical importance:

1) How does adsorption affect flow performance?

2) What is the impact of varying adsorbed layer thickness, pore geometry and compressibility on porosity, permeability, free gas saturation and gas recovery?

3) Which parameters control final recovery?

These questions are answered with analytical relations for recovery and other output; sensitivity analyses on these relations as function of pressure for different pore geometry, compressibility and adsorption layer thickness; and investigating the evolution of shale gas production for such variations.

\section{Mathematical model}

\subsection{Geometry}

Shale gas production from a shale matrix 1D system (e.g., 

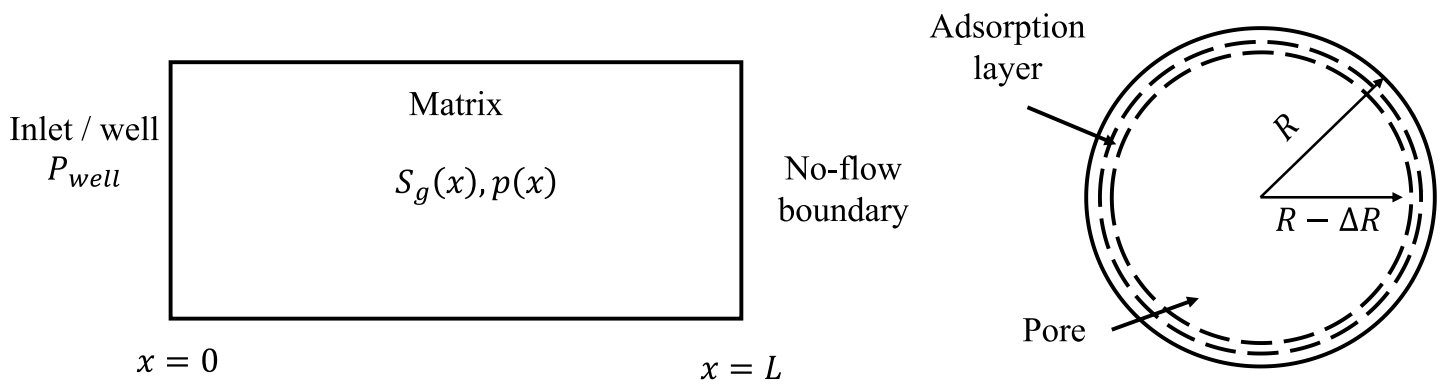

Fig. 1. Geometrical representation of the system. Left: The matrix. Right: Representation of a pore in the matrix.

a core sample or shale connected to a hydraulic fracture) is considered with length $L$, as shown in Fig. 1. Production takes place at $x=0$ with a fixed pressure $P_{\text {well }}$, while there is a noflow boundary at $x=L$ representing the length of the system or half the fracture spacing. The matrix initially contains free and adsorbed gas, with same composition (assumed to be methane). The free gas is compressible, modeled as a real gas, while the adsorbed gas has a constant, relatively high density. No other phases are assumed present. Pores have the shape of an $n$-sphere. This means, for $n=1$ the pores are planar, for $n=2$ cylindrical and for $n=3$ spherical, while intermediate values indicate geometrical configurations in between. In accordance with standard reservoir modeling assumptions, compression is accounted for via changes in the pore volume and a fixed bulk volume is considered.

\subsection{Transport equation}

Performing a mass balance on the system, the change in mass of free and adsorbed gas in a bulk volume $A d x$ is:

$$
\begin{aligned}
& {\left[\phi\left(\rho_{g} S_{g}+\rho_{a} S_{a}\right) d x\right]_{t+\Delta t}-\left[\phi\left(\rho_{g} S_{g}+\rho_{a} S_{a}\right) d x\right]_{t}} \\
& =\left(\rho_{g} u\right)_{x} \Delta t-\left(\rho_{g} u\right)_{x+\Delta x} \Delta t
\end{aligned}
$$

where $\rho_{g}$ and $\rho_{a}$ are free and adsorbed gas densities respectively (in mass per phase volume), $\phi$ is prosity, $S_{g}$ is free phase saturation, $S_{a}$ is adsorbed phase saturation, $t$ is time, $u$ is Darcy free gas velocity and $A$ is the constant cross section area (omitted from the equation). Rearranging and letting $\Delta x$, $\Delta t \rightarrow 0$ gives the following mass conservation equation:

$$
\partial_{t}\left\{\phi\left[\rho_{g} S_{g}+\rho_{a}\left(1-S_{g}\right)\right]\right\}=-\partial_{x}\left(\rho_{g} u\right)
$$

Above, it is used that the two saturations sum to 1 and the adsorbed phase saturation was eliminated. $\rho_{a}$ will be assumed constant (similar to a liquid) while $\rho_{g}$ behaves according to a real gas:

$$
\rho_{g}=\frac{p M}{z R_{g} T}
$$

where $p$ is gas pressure, $R_{g}$ is the gas constant, $T$ is absolute temperature, $M$ is gas molar weight and $z$ is compressibility factor. The Peng and Robinson (1976) equation of state is used to calculate $z$, see Appendix A. In Eq. (2) the gas flux $u$ is described using Darcy's law:

$$
u=-\frac{K_{a}}{\mu} \partial_{x} p
$$

in which $K_{a}$ is pressure-dependent apparent permeability and $\mu$ is pressure-dependent gas viscosity. $\mu(p)$ is calculated using the Lee et al. (1964) correlation, see Appendix B. The above relations lead to the following form of the transport equation:

$$
\partial_{t}\left[\phi\left(\frac{p}{z} S_{g}+R_{g} T \rho_{a} \frac{1-S_{g}}{M}\right)\right]=\partial_{x}\left(\frac{p}{z} \frac{K_{a}}{\mu} \partial_{x} p\right)
$$

\subsection{Porosity model accounting for pore geometry and adsorption}

The $n$-volume $V_{n}$ of an $n$-sphere pore with radius $R$ is (Evans, 1947):

$$
V_{n}=\frac{\pi^{\frac{n}{2}}}{\Gamma\left(\frac{n}{2}+1\right)} R^{n}
$$

The Gamma function $(\Gamma)$ above is defined as a semi-infinite integral:

$$
\Gamma(m)=\int_{x=0}^{\infty} x^{m-1} e^{-x} d x
$$

which is corresponding to the factorial function as below for integer input values:

$$
\Gamma(m)=(m-1) !
$$

By considering an $n$-sphere, the $n$-volume will be obtained. To turn this into a three-dimensional volume a factor $C$ (volume per $n$-volume) was used. For $n=1, C$ equals the cross section of a fracture, for $n=2$ it is the length of a cylinder, and for $n=3$ it equals 1 . Let $N$ be the total number of pores per unit bulk volume. The total pore volume $V_{p}$ is then:

$$
V_{p}=N C V_{n}
$$

Porosity follows as:

$$
\phi=\frac{V_{p}}{V_{b}}=\frac{N C}{V_{b}} V_{n}
$$

Assuming the porosity is known at initial time $\phi_{0}$, then:

$$
\phi_{0}=\frac{N C}{V_{b}} V_{n}^{0}
$$

where $V_{b}$ is bulk volume, $V_{n}^{0}$ is the $n$-volume of a pore at initial state. The ratio $N C / V_{b}$ may be determined from Eq. (11) and substituted into Eq. (10) to yield porosity written as:

$$
\phi=\phi_{0} \frac{V_{n}}{V_{n}^{0}}=\phi_{0}\left(\frac{R}{R_{0}}\right)^{n}
$$


where $R$ is pore radius, $R_{0}$ is initial pore radius. These relations can then be used to equivalently express pore radius as function of porosity given the initial radius and porosity:

$$
R=R_{0}\left(\frac{\phi}{\phi_{0}}\right)^{\frac{1}{n}}
$$

Porosity may be taken as a function of pressure and (pore) compressibility $c_{k}$ :

$$
\phi=\phi_{0} \exp \left[c_{k}\left(p-p_{0}\right)\right]
$$

where $p_{0}$ is the initial pressure, corresponding to the initial porosity $\phi_{0}$ and radius $R_{0}$. The amount of adsorbed gas in shale is commonly described by the Langmuir isotherm which assumes gas attaches to the surface as a single layer (a monolayer). Regardless of pore geometry, an adsorbed layer in the pores with thickness $\Delta R$ following a Langmuir relation with pressure may be defined as (Xiong et al., 2012; Jiang and Yang, 2018):

$$
\Delta R=\Delta R_{\max } \frac{p}{p_{L}+p}
$$

where $p_{L}$ is the Langmuir pressure, $\Delta R_{\max }$ is the maximum thickness at theoretically infinite pressure the thickness is half that value at the Langmuir pressure $p_{L}$. By representing the adsorbed layer by an isotherm it is implicitly assumed that the adsorption process occurs instantaneously. Adsorption can occur at a finite rate where a source term is better suited to represent the process. In that case, the thickness $\Delta R$ would be a function of both pressure and time and reach the equilibrium state Eq. (15) only when sufficient time had passed.

For a given layer thickness $\Delta R$ in a pore, using Eqs. (6) and (9), the volume of free gas $V_{g}$ in the pore volume is:

$$
\begin{gathered}
V_{g}=N C \frac{\pi^{\frac{n}{2}}}{\Gamma\left(\frac{n}{2}+1\right)} R_{e}^{n} \\
R_{e}=R-\Delta R
\end{gathered}
$$

where $R_{e}$ is the effective radius. The free gas saturation $S_{g}$ is defined as the fraction of pore volume filled by free gas. The remaining fraction is adsorbed gas.

$$
S_{g}=\frac{V_{g}}{V_{p}}=\left(\frac{R_{e}}{R}\right)^{n}
$$

This can also be used to define an effective porosity $\left(\phi_{e}\right)$, i.e., the volume fraction of the bulk volume occupied by free gas:

$$
\phi_{e}=\phi S_{g}=\phi_{0}\left(\frac{R}{R_{0}}\right)^{n}\left(\frac{R_{e}}{R}\right)^{n}=\phi_{0}\left(\frac{R_{e}}{R_{0}}\right)^{n}
$$

At initial state it holds that:

$$
\phi_{e 0}=\phi_{0}\left(\frac{R_{e 0}}{R_{0}}\right)^{n}
$$

Combining the two Eqs. (19) and (20) results in:

$$
\phi_{e}=\phi_{e 0}\left(\frac{R_{e}}{R_{e 0}}\right)^{n}
$$

\subsection{Apparent permeability model accounting for pore geometry and adsorption}

Apparent permeability is typically separated into two factors, where the first is the intrinsic permeability $k_{\infty}$ and the second is the slip correction factor $f$, typically modeled as dependent on the Knudsen number $K_{n}$ :

$$
K_{a}=k_{\infty} f\left(K_{n}\right)
$$

\subsubsection{Intrinsic permeability model}

A similar derivation will be followed as Zimmermann and Bodvarsson (1996) did for a plane fracture $(n=1)$. Consider flow through a pore with geometry characterized by the effective radius $R_{e}$ and parameter $n$. The interstitial velocity field in the pore is denoted $\mathbf{v}(r, x, t)=\left(v_{r}(r, x, t), v_{x}(r, x, t)\right)$ where it is assumed that the field can depend on the radial distance $r$ from the pore center, the position along the general flow axis $x$ and time $t . r$ is normal to $x$. The evolution of the velocity field is given by Navier-Stokes equation (Batchelor and Batchelor, 2000):

$$
\partial_{t} \mathbf{v}+(\mathbf{v} \cdot \nabla) \mathbf{v}=\mathbf{F}-\frac{1}{\rho} \nabla p+\frac{\mu}{\rho} \nabla^{2} \mathbf{v}
$$

where $\mathbf{F}$ is a body force (gravity) and $p(x, w, t)$ is the pressure field. Steady state is assumed and gravity ignored such that $\partial_{t} u=0$ and $\mathbf{F}=0$ which yields:

$$
\mu \nabla^{2} \mathbf{v}-\rho(\mathbf{v} \cdot \nabla) \mathbf{v}=\nabla p
$$

Since permeability is an intrinsic property, flow conditions can be based on an incompressible fluid, which has the property:

$$
\nabla \cdot \mathbf{v}=0
$$

Gas compressibility and slip will be accounted for as separate mechanisms. Three equations then result for the two velocity components $v_{r}, v_{x}$ and the pressure $p$. The flow is considered to be in one direction $x$, such that the radial velocity component is zero, $v_{r}=0$ while the velocity component in $x$-direction $v_{x}$ will vary with $r$ only: $v_{x}=v_{x}(r)$. The incompressibility condition is then obeyed:

$$
\nabla \cdot \mathbf{v}=\left(\partial_{r}, \partial_{x}\right) \cdot\left(v_{r}, v_{x}\right)=\partial_{r} v_{r}+\partial_{x} v_{x}=0
$$

Also, the pressure gradient components are considered a constant in $x$-direction and zero in $r$-direction, respectively:

$$
\nabla p=\left(\partial_{r} p, \partial_{x} p\right)=\left(0, \partial_{x} p\right)
$$

The differential terms in Eq. (24) turn into:

$$
\begin{aligned}
(\mathbf{v} \cdot \nabla) \mathbf{v} & =\left(v_{r} \partial_{r}+v_{x} \partial_{x}\right)\left(v_{r}, v_{x}\right) \\
= & \left(v_{r} \partial_{r} v_{r}+v_{x} \partial_{x} v_{r}, v_{r} \partial_{r} v_{x}+v_{x} \partial_{x} v_{x}\right)=(0,0) \\
\nabla^{2} \mathbf{v} & =\left\{0, \frac{1}{r^{n-1}} \partial_{r}\left[r^{n-1} \partial_{r} v_{x}(r)\right]+\partial_{x x} v_{x}(r)\right\} \\
& =\left\{0, \frac{1}{r^{n-1}} \partial_{r}\left[r^{n-1} \partial_{r} u_{x}(r)\right]\right\}
\end{aligned}
$$

The following equation is then obtained for the velocity component $v_{x}(r)$ : 


$$
\mu \frac{1}{r^{n-1}} \partial_{r}\left[r^{n-1} \partial_{r} v_{x}(r)\right]=\partial_{x} p
$$

Integrating the equation once gives:

$$
\partial_{r} v_{x}(r)=\frac{1}{r^{n-1}}\left(\frac{1}{n} \frac{\partial_{x} p}{\mu} r^{n}+r\right)
$$

where $Y$ is an integration constant. It is assumed the profile is symmetric such that $\left.\partial_{r} v_{x}\right|_{r=0}=0$ :

$$
\left.\partial_{r} v_{x}\right|_{r=0}=\left.\Upsilon r^{-(n-1)}\right|_{r=0}=0
$$

Since $n \geq 1$ the above equality can only hold if $r=0$. Eq. (31) thus simplifies to:

$$
\partial_{r} v_{x}(r)=\frac{1}{n} \frac{\partial_{x} p}{\mu} r
$$

Integrating again results in:

$$
v_{x}(r)=-\frac{1}{n} \frac{\partial_{x} p}{\mu} \frac{1}{2}\left(R_{e}^{2}-r^{2}\right)
$$

During the integration a no-flow condition was applied at $r=R_{e}$, the outermost radius accessible to gas flow, i.e., $v_{x}\left(r=R_{e}\right)=0$. Implicitly the adsorbed phase is assumed immobile. As a remark, note that surface diffusion, the movement of adsorbed phase due to a gradient in adsorbed phase concentration, has been considered important in some works (Yang et al., 2016; Ren et al., 2017; Yin et al., 2017), but is not accounted for here.

Assume that the pore has an $n-1$-area $A_{n-1}$ (with unit $m^{n-1}$ ) when the pore has $n$-volume $V_{n}$ (with unit $m^{n}$ ) which depend on $n$ and the effective radius $R_{e}$ as follows:

$$
\begin{aligned}
A_{n-1}\left(R_{e}\right) & =\frac{2 \pi^{\frac{n}{2}}}{\Gamma\left(\frac{n}{2}\right)} R_{e}^{n-1} \\
V_{n}\left(R_{e}\right) & =\frac{\pi^{\frac{n}{2}}}{\Gamma\left(\frac{n}{2}+1\right)} R_{e}^{n}
\end{aligned}
$$

The total flow rate $Q$ can be calculated by considering the contributions of flow through all $n$-volume elements $d V_{n}$ in the pore. The incremental change in $V_{n}$ given a change in radius for an arbitrary value $r$ is related as:

$$
d V_{n}(r)=A_{n-1}(r) d r
$$

The cumulative flow is found by integrating the flow through every element of the entire cross section:

$$
\begin{gathered}
Q=\int_{r=0}^{R_{e}} v_{x}(r) C_{f} A_{n-1} d r \\
=-\frac{C_{f}}{2 n} \frac{\partial_{x} p}{\mu} \frac{2 \pi^{\frac{n}{2}}}{\Gamma\left(\frac{n}{2}\right)} \int_{r=0}^{R_{e}}\left(R_{e}^{2} r^{n-1}-r^{n+1}\right) d r \\
=-C_{f} \frac{\partial_{x} p}{\mu} \frac{2 \pi^{\frac{n}{2}}}{\Gamma\left(\frac{n}{2}\right)} \frac{R_{e}^{n+2}}{n^{2}(n+2)}
\end{gathered}
$$

where the expressions for the flow profile $v_{x}(r)$ and the $n-1$-area $A_{n-1}$ from Eqs. (34) and (35) have been inserted, respectively. The effective cross section open for gas flow is:

$$
\begin{aligned}
A \phi_{e} & =\int_{r=0}^{R_{e}} C_{f} \frac{d V_{n}}{d r} d r=C_{f} V_{n}\left(R_{e}\right) \\
& =C_{f} \frac{\pi^{\frac{n}{2}}}{\Gamma\left(\frac{n}{2}+1\right)} R_{e}^{n}
\end{aligned}
$$

The average interstitial velocity $v$ in $x$-direction is found by dividing the total flow rate by the porous cross section and the tortuosity $\tau$ (accounting for the fact that flow may not be along a straight line).

$$
\begin{aligned}
v=\frac{Q}{A \phi_{e} \tau} & =-\frac{C_{f} \frac{\partial_{x} p}{\mu} \frac{2 \pi^{\frac{n}{2}}}{\Gamma\left(\frac{n}{2}\right)} \frac{R_{e}^{n+2}}{n^{2}(n+2)}}{C_{f} \frac{\pi^{\frac{n}{2}}}{\Gamma\left(\frac{n}{2}+1\right)} R_{e}^{n} \tau} \\
& =-\frac{\partial_{x} p}{\tau \mu} \frac{R_{e}^{2}}{n(n+2)}
\end{aligned}
$$

The Darcy velocity equals the product of interstitial velocity and effective porosity:

$$
u=\phi_{e} v=-\frac{\phi_{e}}{\tau \mu} \frac{R_{e}^{2}}{n(n+2)} \partial_{x} p
$$

This can be compared with Darcy's law which states (without consideration of slip):

$$
u=-\frac{k_{\infty}}{\mu} \partial_{x} p
$$

This implies that the intrinsic permeability $k_{\infty}$ for the different geometries can be expressed as:

$$
k_{\infty}=\frac{\phi_{e}}{\tau} \frac{R_{e}^{2}}{n(n+2)}
$$

Tortuosity is often correlated with porosity, as reviewed by Shen and Chen (2007). A tortuosity relation with effective porosity is applied using the correlation by Bruggemann (1935):

$$
\tau=\phi_{e}^{-0.25}
$$

As special cases of the derived model for intrinsic permeability $k_{\infty}$, note that $n=1$ gives planar fracture pores, and $n=2$ gives cylindrical pores. The corresponding $n$-volumes and $n-1$-areas from Eq. (35) are then:

$$
\begin{gathered}
A_{0}\left(R_{e}\right)=2, \quad V_{1}\left(R_{e}\right)=2 R_{e}, \quad(n=1) \\
A_{1}\left(R_{e}\right)=2 \pi R_{e}, \quad V_{2}\left(R_{e}\right)=\pi R_{e}^{2}, \quad(n=2)
\end{gathered}
$$

For $n=2, V_{2}$ already corresponds to the cross section of the pore, while for $n=1$ the factor $C_{f}$ should equal the height $h$ of the fracture to give the cross section. The permeability expressions become:

$$
\begin{gathered}
k_{\infty}=\frac{\phi_{e}}{\tau} \frac{R_{e}^{2}}{3}=\frac{\phi_{e}}{\tau} \frac{w_{e}^{2}}{12}, \quad(n=1) \\
k_{\infty}=\frac{\phi_{e}}{\tau} \frac{R_{e}^{2}}{8}, \quad(n=2)
\end{gathered}
$$


where $w_{e}=2 R_{e}$ is the width of the fracture. Both these expressions are consistent with the literature (Carman, 1956; Zimmermann and Bodvarsson, 1996; Civan, 2007), although they typically do not account for the influence of adsorption and thus use the full porosity $\phi$ and radius $R$. Finally, by using the relations between effective radius and effective porosity from Eq. (21) and between tortuosity and effective porosity from Eq. (43), the relation Eq. (42) can be written as:

$$
\frac{k_{\infty}}{k_{\infty 0}}=\left(\frac{R_{e}}{R_{e 0}}\right)^{2+1.25 n}=\left(\frac{\phi_{e}}{\phi_{e 0}}\right)^{1.25+\frac{2}{n}}
$$

\subsubsection{Slip correction factor}

To classify flow in small pores, the dimensionless Knudsen number is commonly used. It is defined as the ratio of molecular mean free path $\lambda$ (Loeb, 2004) to a characteristic pore dimension, in this work assumed to be the effective radius $R_{e}$ :

$$
K_{n}=\frac{\lambda}{R_{e}}=\frac{z \mu}{p R_{e}} \sqrt{\frac{\pi R_{g} T}{2 M}}
$$

Apparent permeability $K_{a}$ is related to intrinsic permeability $k_{\infty}$ according to the Knudsen number relation derived by Beskok and Karniadakis (1999):

$$
\begin{aligned}
K_{a} & =k_{\infty}\left[1+\alpha\left(K_{n}\right) K_{n}\right]\left(1+\frac{4 K_{n}}{1+K_{n}}\right) \\
\alpha\left(K_{n}\right) & =\frac{128}{15 \pi^{2}} \tan ^{-1}\left(4 K_{n}^{0.4}\right)
\end{aligned}
$$

\subsection{Initial and boundary conditions}

The system has a defined initial pressure $p_{0}$ which also defines the initial radius, porosity, adsorption layer thickness and saturation:

$$
\begin{gathered}
p(x, t=0)=p_{0}, \quad R(x, t=0)=R\left(p_{0}\right) \\
\Delta R(x, t=0)=\Delta R\left(p_{0}\right), \quad S_{g}(x, t=0)=S_{g}\left(p_{0}\right)
\end{gathered}
$$

The boundary at $x=0$ is open with a fixed pressure $p_{\text {well }}$ while a closed boundary is applied at $x=L$ :

$$
p(x=0, t)=p_{\text {well }}, \quad\left(\partial_{x} p\right)_{x=L}=0
$$

\subsection{Original and current gas in place and gas recovery}

The conserved property $M_{T}$ in the transport equation (Eq. (5)) consists of a free gas term $M_{1}$ and an adsorbed gas term $M_{2}$, both in units of pressure due to scaling constant properties:

$$
M_{1}=\phi \frac{p S_{g}}{z}, \quad M_{2}=\phi \frac{R_{g} T \rho_{a}}{M}\left(1-S_{g}\right), \quad M_{T}=M_{1}+M_{2}
$$

Gas currently in place $\left(G_{C I P}\right.$ with units of length times pressure) can be evaluated by integrating over the system. Similarly, gas initially in place $G_{I I P}$ is $G_{C I P}$ uniformly evaluated at the initial pressure $p_{0}$ :

$$
\begin{aligned}
G_{C I P} & =\int_{x=0}^{L}\left(M_{1}+M_{2}\right) d x \\
G_{I I P} & =G_{C I P}\left(p_{0}\right)=L\left[M_{1}\left(p_{0}\right)+M_{2}\left(p_{0}\right)\right]
\end{aligned}
$$

Gas recovery factor $R F$ is the fraction of $G_{I I P}$ that has been produced:

$$
R F=1-\frac{G_{C I P}}{G_{I I P}}
$$

It was shown by Andersen (2021a), for a similar system, that if the conserved property $M_{T}$ is monotonous with pressure (which follows since higher pressure leads to storing more gas), the system can be expressed as a nonlinear diffusion equation with $M_{T}$ as the independent variable. It was demonstrated theoretically that recovery $R F$ against time then is proportional to the square root of time until the no-flow boundary is encountered.

If a given (well) pressure is allowed to become uniform, the highest recovery possible at that state is obtained, in terms of original gas in place $O_{G I P}$, called the ultimate recovery factor $R F_{\infty}(p)$ :

$$
\begin{aligned}
R F_{\infty}(p) & =1-\frac{G_{C I P}(p)}{O_{G I P}} \\
& =1-\frac{\phi(p)\left\{\frac{p S_{g}(p)}{z(p)}+\frac{R_{g} T \rho_{a}}{M}\left[1-S_{g}(p)\right]\right\}}{\phi_{0}\left[\frac{p_{0} S_{g 0}}{z_{0}}+\frac{R_{g} T \rho_{a}}{M}\left(1-S_{g 0}\right)\right]}
\end{aligned}
$$

Calculations of other average properties are shown in Appendix C. In the limit where the pressure goes to zero, the adsorbed layer thickness goes to zero, see Eq. (15), while pore radius stays finite, thus saturation goes to 1 and recovery goes to 1. For the special case where adsorption is negligible (na), i.e., $S_{g}=1$, and gas is ideal $z=1$, recovery is given by:

$$
R F_{\infty}^{n a}(p)=1-\frac{p}{p_{0}} \frac{\phi(p)}{\phi_{0}}
$$

If also pore compressibility is negligible $(n a, n c)$ :

$$
R F_{\infty}^{n a, n c}(p)=1-\frac{p}{p_{0}}
$$

demonstrating a linear relation between recovery and pressure.

\subsection{Implementation}

The final model consists of the transport Eq. (5), coupled with the relations for porosity (Eq. (14)), viscosity (Eq. (B1)) gas compressibility factor (Eq. (A-4)), radius (Eq. (13)), effective radius (Eq. (17)), intrinsic permeability (Eq. (42)), and apparent permeability (Eq. (50)). The system was solved by operator splitting into a 'Free gas transport' system with fixed-in-time spatial distributions of porosity, saturation and permeability, and a 'Redistribution' system where these properties were updated locally to satisfy the constraint equations and preservation of mass. The simulation time $T_{\text {total }}$ was divided into equal splitting time steps $\Delta T$; the time running 'Free gas transport' before a 'Redistribution'. Appendix D provides the details and discretization for this process. The numerical procedure was implemented in MATLAB. The $x$ axis was discretized into 50 equal cells and $\Delta T$ was selected to $12 \mathrm{hrs}$, which was sufficient for convergence, see Appendix E. Numerical validation against a commercial software is presented in Appendix E. 
Table 1. Input parameters used for reference case simulations.

\begin{tabular}{|c|c|c|}
\hline Constant parameters & Value & Units \\
\hline$*$ Well pressure, $p_{\text {well }}$ & $2.75 \times 10^{6}$ & $\mathrm{~Pa}$ \\
\hline *Initial pressure, $p_{0}$ & $27.5 \times 10^{6}$ & $\mathrm{~Pa}$ \\
\hline Gas constant, $R_{g}$ & 8.314 & $\mathrm{~J} / \mathrm{mol} \cdot \mathrm{K}$ \\
\hline Matrix length, $L$ & 10 & $\mathrm{~m}$ \\
\hline Initial pore radius, $R_{0}$ & 4 & $\mathrm{~nm}$ \\
\hline *Temperature, $T$ & 355.37 & $\mathrm{~K}$ \\
\hline Initial tortuosity, $\tau_{0}$ & 2.197 & - \\
\hline Gas molar weight, $M$ & $16.04 \times 10^{-3}$ & $\mathrm{~kg} / \mathrm{mol}$ \\
\hline *Initial porosity, $\phi_{0}$ & 0.06 & - \\
\hline$*$ Ads. gas density, $\rho_{a}$ & 343.8 & $\mathrm{~kg} / \mathrm{m}^{3}$ \\
\hline *Langmuir pressure, $p_{L}$ & $8.11 \times 10^{6}$ & $\mathrm{~Pa}$ \\
\hline Varied parameters (base value) & Value & Units \\
\hline$*$ Pore compressibility, $c_{k}$ & $1.55 \times 10^{-9}$ & $1 / \mathrm{Pa}$ \\
\hline Geometry parameter, $n$ & 2 & - \\
\hline Max ads. thickness, $\Delta R_{\max }$ & 0.8 & $\mathrm{~nm}$ \\
\hline *Langmuir volume, $V_{L}$ & 0.2338 & $\mathrm{mmol} / \mathrm{g}$ \\
\hline Numerical parameters & Value & Units \\
\hline Grid Cells, $N_{x}$ & 50 & - \\
\hline Splitting step, $\Delta T$ & 12 & hrs. \\
\hline Simulation time, $T_{\text {total }}$ & 30000 & $\mathrm{~d}$ \\
\hline
\end{tabular}

\section{Results and discussion}

\subsection{Model input}

The model input reference parameters are shown in Table 1. They are taken from Pang et al. (2017) who quantified the impact of pore compressibility and adsorption on porosity based on experiments on shale from the Yanchang formation in the Ordos basin. A fixed adsorbed phase density is assumed of $343.8 \mathrm{~kg} / \mathrm{m}^{3}$, as reported by Pang et al. (2017). Perez and Devegowda (2017) obtained a similar value from molecular simulations, while Chen et al. (2019) reported a wider range $\left(150-660 \mathrm{~kg} / \mathrm{m}^{3}\right)$, but at more varying experimental conditions. Reference $\Delta R_{\max }$ was selected as $0.8 \mathrm{~nm}$, and low and high values were taken as 0.4 and $2 \mathrm{~nm}$. The low to high values of $\Delta R_{\max }$ corresponded to initial mass fractions of adsorbed gas $\left(M_{2}^{0} / M_{T}^{0}\right)$ of $0.27,0.47$ and 0.78 , similar to observations in the literature; 20\% to 85\% (Montgomery et al., 2005; Ambrose et al., 2012). The $\Delta R_{\max }$ range is also comparable to measured methane adsorption thicknesses between 0.25 and $1.5 \mathrm{~nm}$ reported by Chen et al. (2019) for Longmaxi shale and the molecular diameter of methane which is $0.38 \mathrm{~nm}$, often applied as monolayer adsorption thickness. Reference $n$ was set to 2 to indicate cylindrical pores. The match between our

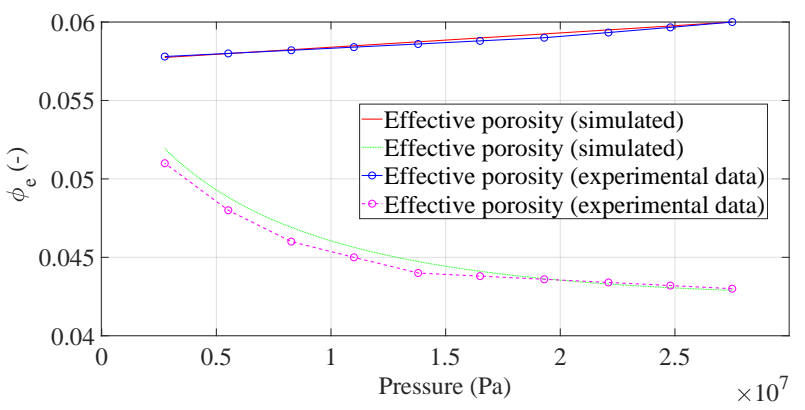

Fig. 2. Comparison of experimental effective porosity against pressure from Pang et al. (2017) and our model. The top curves show porosity reduction in presence of non-adsorbing gas, while the lower curves show porosity change when adsorption occupies a significant amount of the pore space.

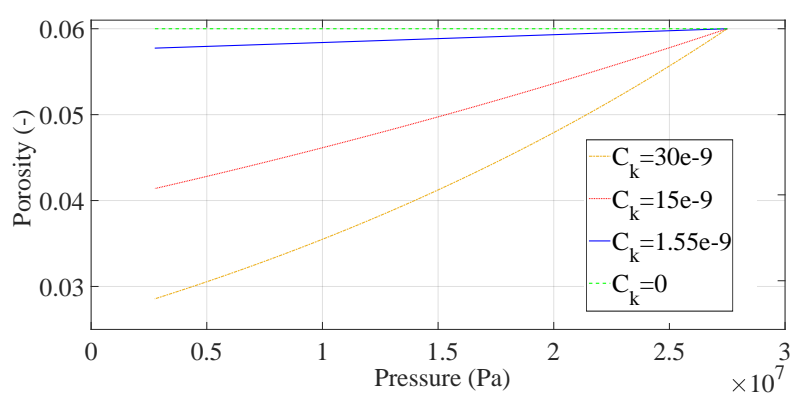

Fig. 3. Porosity as function of pressure for different compressibilities (in units of $\mathrm{Pa}^{-1}$ ).

model and the data from Pang et al. (2017) is shown in Fig. 2, where the pore compressibility captures the decline in porosity on pressure depletion with non-adsorbing gas present, and adding the adsorption model captures how porosity actually increases from a low value on pressure depletion since desorption opens the pore space.

In the following, unless otherwise stated, all parameters take reference values. The initial porosity $\phi_{0}$ and pore radius $R_{0}$ are set independently and the same in all example cases, but once set, they vary dependently. As indicated in the table, the main varied parameters are the pore compressibility $c_{k}$, the geometry parameter $n$ and the max adsorption layer thickness $\Delta R_{\max }$. The initial pore volume will be the same in all cases, but it may vary how much is initially filled by adsorbed phase.

\subsection{Static model behavior}

\subsubsection{Porosity relations}

Fig. 3 shows the effect of compressibility on porosity. Higher compressibility results in greater porosity decline for a given pressure depletion. The reference compressibility of $1.55 \times 10^{-9} \mathrm{~Pa}^{-1}$ from Pang et al. (2017) reduces the porosity from 0.06 to 0.0575 . For illustration this and higher values will be used to show how compressibility and adsorption interact, but it is noted that shales such as Marcellus, Barnett and Chinsui have lower compressibility around $0.1-0.8 \times 10^{-9}$ $\mathrm{Pa}^{-1}$ (Dong et al., 2010; Yu and Sepehrnoori, 2014). 

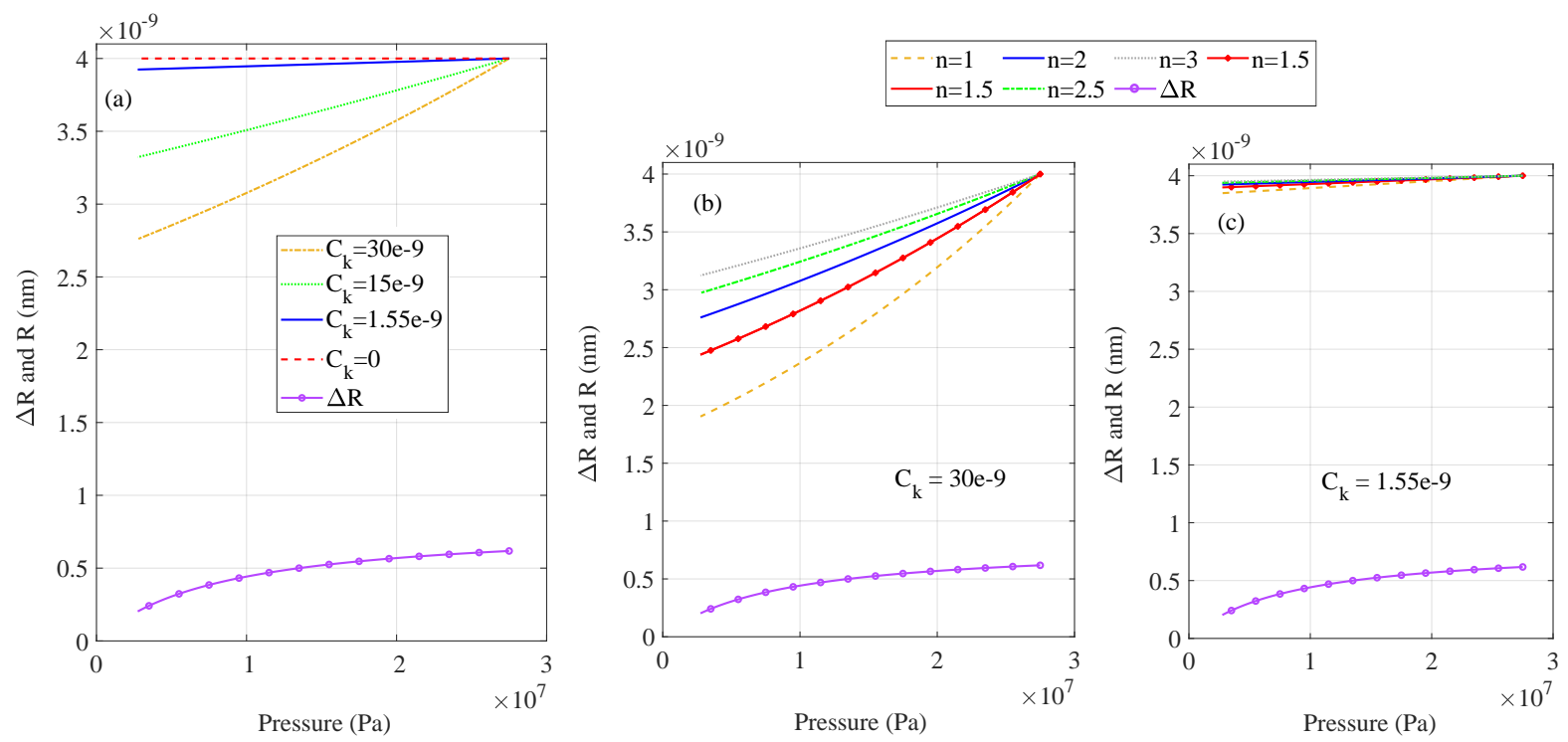

Fig. 4. Adsorbed layer thickness $\Delta R(p)$ and pore radius $R(p)$ vs pressure for different values of compressibility (in $\left.\mathrm{Pa}^{-1}\right)(\mathrm{a})$, and variations of geometry parameter $n$ for a high compressibility (b) and low compressibility (c).
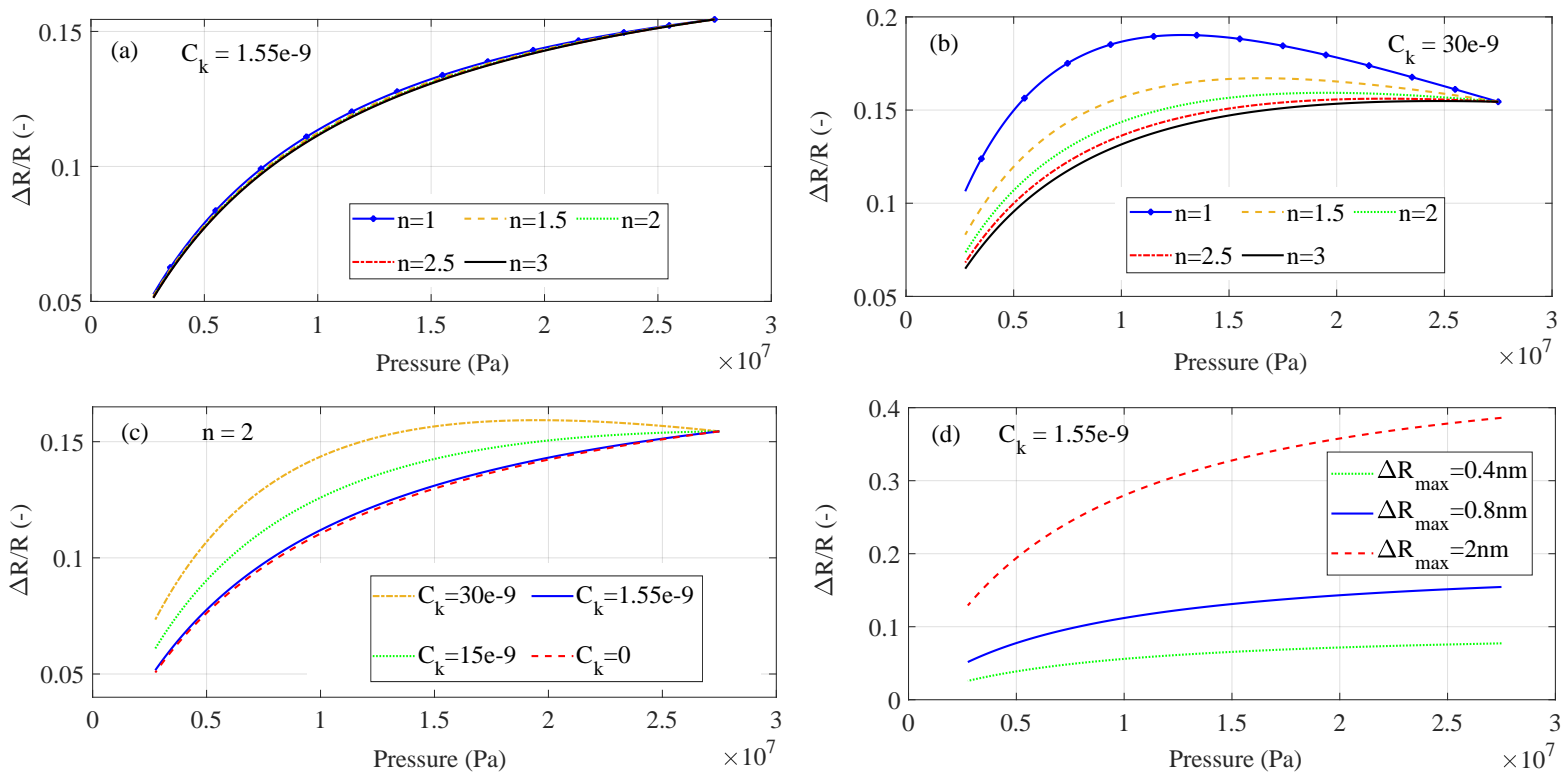

Fig. 5. The fraction $\Delta R / R$ vs pressure for variations of geometry parameter $n$ for a low compressibility (a) and high compressibility (b), variation of compressibility (c) and $\Delta R_{\max }$ (d). Note that the $y$-axis scales are not identical.

\subsubsection{Pore radius and adsorption layer}

In Fig. 4(a) it is observed that higher compressibility results in greater reduction of pore radius at a given pressure, which follows from Eqs. (13) and (14). In Fig. 4(b), $n$ is varied using the base compressibility $1.55 \times 10^{-9} \mathrm{~Pa}^{-1}$. The small changes of $R$ with $n$ reflect that the radius (and porosity) stays near their original values. Changes from compressibility are then small irrespective of geometrical differences. When a high compressibility of $30 \times 10^{-10} \mathrm{~Pa}^{-1}$ is used in Fig. 4(c), the radius reduces and varies more with $n$. The radius reduces the most when $n$ is low. In other words, the same reduction in porosity results in greater reduction of pore 'radius' when the pores are more planar ( $n$ close to 1$)$ than spherical ( $n$ close to 3). The adsorption layer thickness is defined uniquely by pressure according to the Langmuir relation (Eq. (15)) and does not change with $c_{k}$ or $n$. Radius appears to change close to linearly with pressure for different $n$ and $c_{k}$, while layer thickness changes nonlinearly: Slowly at high pressures and more rapidly at low pressures.

The fraction $\Delta R / R$ is plotted vs pressure for variations of geometry parameter $n$ with low and high compressibility (Figs. 5(a) and 5(b), respectively), variation of compressibility $c_{k}$ (Fig. 5(c)), and $\Delta R_{\max }$ (Fig. 5(d)). From the above considerations, for a given $n$ (geometry) the layer fills a greater fraction $\Delta R / R$ with a higher compressibility than a low. And for a given compressibility, the layer fills a greater fraction $\Delta R / R$ 

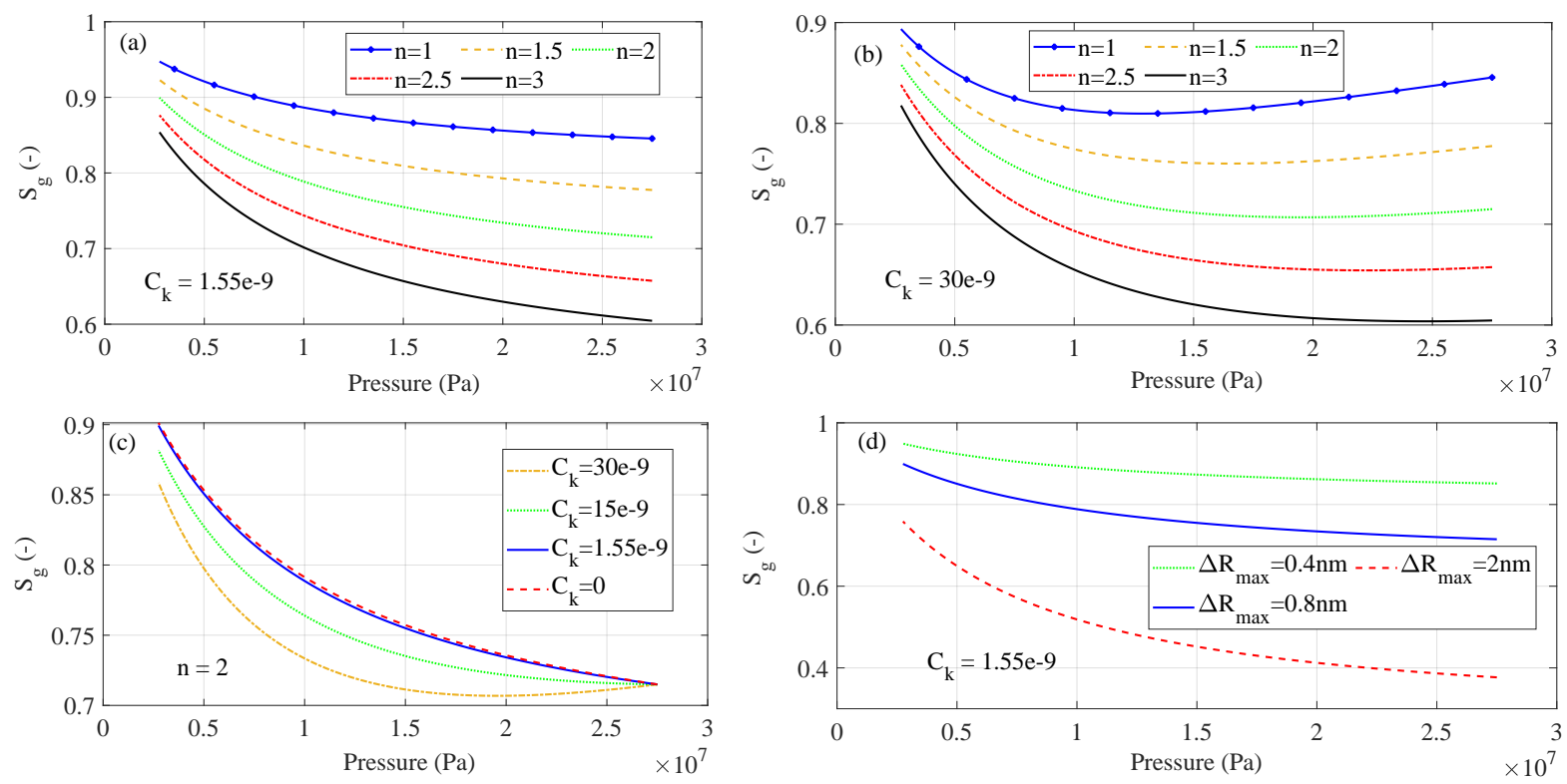

Fig. 6. Free gas saturation $S_{g}$ vs pressure for variations of geometry parameter $n$ for a low compressibility (a) and high compressibility (b), variation of compressibility (c) and $\Delta R_{\max }$ (d). Note that the $y$-axis scales are not identical.

for a low $n$ than a high $n$.

The fraction $\Delta R / R$ reduces towards low pressures for small changes in pore radius and greater reduction in adsorbed layer thickness. At zero compressibility (red curve in Fig. 5(c)) the radius is constant, while for more compressible cases, the radius changes linearly. As long as the radius changes little compared to the initial radius, the behavior is comparable to the incompressible case. For high compressibilities the pore radius can reduce more rapidly than the adsorbed layer at high pressures, but slower at low pressures. There is then a peak in how much the layer occupies of the pore radius at intermediate pressures (orange line in Fig. 5(c)). The effect of geometry comes more into play in highly compressible shale, see Fig. 5(b). High $n$ maintains higher pore radius with pressure depletion, and thus the adsorbed layer fills a lower fraction of the radius at low pressure than low $n$ cases. When maximum adsorption layer thickness is increased the fraction $\Delta R / R$ is generally increased, see Fig. 5(d).

\subsubsection{Free gas saturation}

Fig. 6 plots free gas saturation $S_{g}$ as function of pressure using Eq. (18) for the aforementioned cases. The free gas saturation naturally has an opposite trend with pressure as the fraction $\Delta R / R$ but the relation is nonlinear according to the exponent $n$, see Eq. (18).

Pressure depletion reduces the fraction $\Delta R / R$ for low compressible cases which causes gas saturation to increase. Increased compressibility (Fig. 6(c)) increases the fraction $\Delta R / R$ and hence lowers the free gas saturation systematically. $n$ not only affects the radius, but also influences how saturation is related to $\Delta R / R$. For example, in a round pore (high $n$ ) the same adsorbed layer thickness $\Delta R$ takes more of the volume than in a fracture-like pore (low $n$ ) for same porosity since the layer covers the radial interval taking most volume. Hence, for increased $n$ the free gas saturation reduces quickly.
Especially, the relations $\Delta R / R$ which were invariant with $n$ at low compressibility (Fig. 5(a)) and varying little with high compressibility (Fig. 5(b)), are equivalent to saturation relations that vary significantly with $n$, see Figs. 6(a) and 6(b). The reduction of saturation with $n$ includes the initial saturation which is natural since the radius and layer thickness are defined by the pressure, while the volume fraction depends on the pore geometry. Finally, increased $\Delta R_{\max }$ reduces the free gas saturation (Fig. 6(d)).

\subsubsection{Effective porosity}

The effective porosity, which measures the volume fraction occupied by free gas, is shown in Fig. 7. In low compressible cases the desorption causes it to increase with pressure depletion, while in compressible dominated cases it can reduce. High $n$ cases have the lowest effective porosity, with clear impact also for low compressibility cases.

\subsubsection{Gas recovery}

Recovery is shown vs pressure based on Eq. (57) in Fig. 8. Higher $c_{k}$ (Fig. 8(c)) reduces the pore volume more at a given pressure and yields higher recovery. Although the adsorbed layer thickness is a unique function of pressure, the amount free gas is proportional to the remaining pore volume, which is reduced. Hence, recovery increases with compressibility.

Adsorbed gas is not produced significantly until low pressures. Recovery therefore generally decreases when the adsorbed fraction increases, e.g., when $\Delta R_{\max }$ increases (Fig. $8(\mathrm{~d})$ ). More spherical pores (higher $n$ ) have a higher volume content of adsorbed gas (lower gas saturation, see Figs. 6(a) and $6(\mathrm{~b}))$. The adsorbed mass fraction is even higher due to the density. Higher $n$ therefore also results in lower recovery (Figs. 8(a) and 8(b)) at a given pressure.

For low $n$, a lower fraction gas is in adsorbed form (Figs. 6(a) and 6(b)). If porosity does not vary greatly and the 

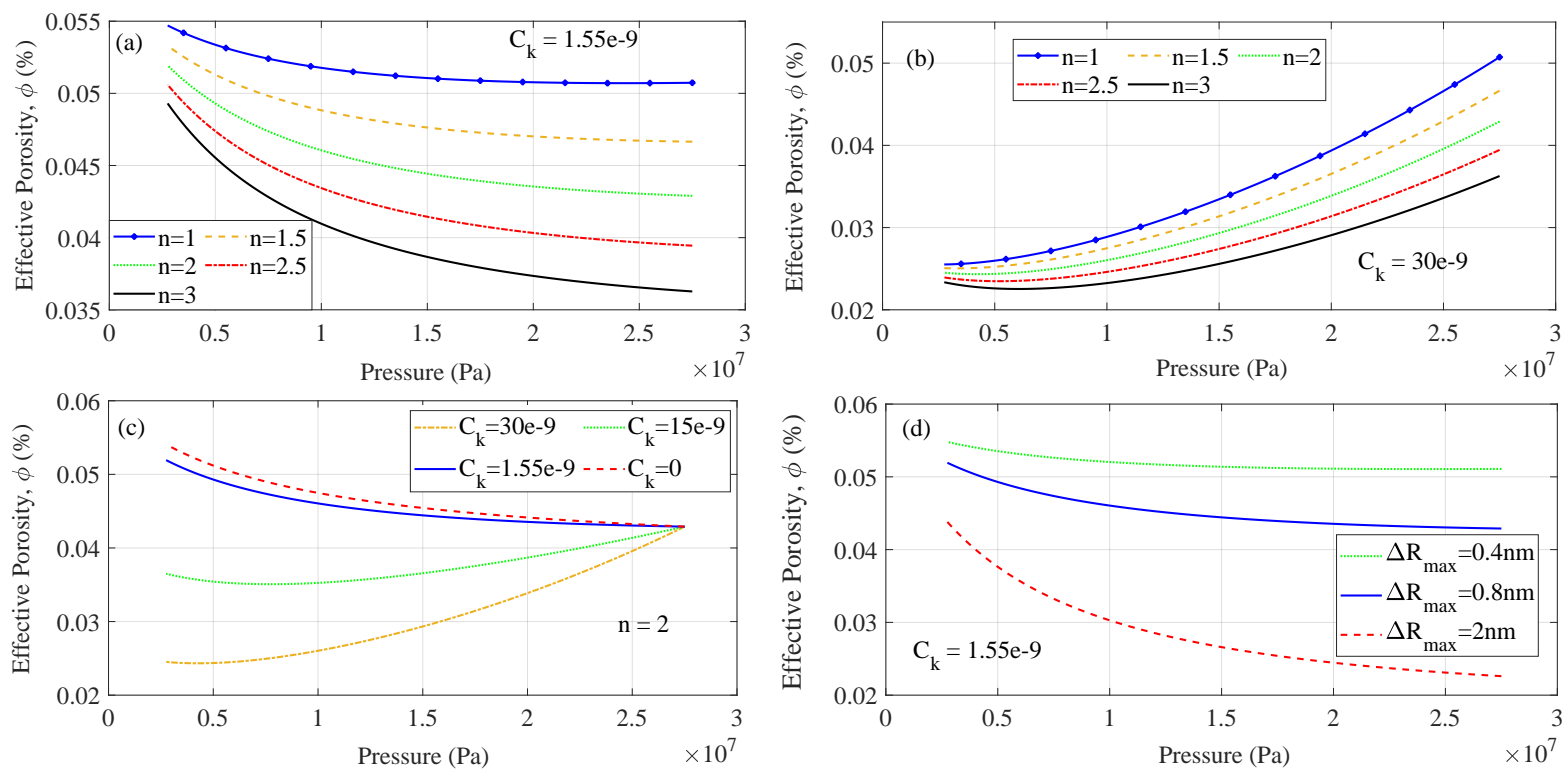

Fig. 7. Effective porosity $\phi_{e}$ vs pressure for variations of geometry parameter $n$ for a low compressibility (a) and high compressibility (b), variation of compressibility (c) and $\Delta R_{\max }(\mathrm{d})$. Note that the $y$-axis scales are not identical.
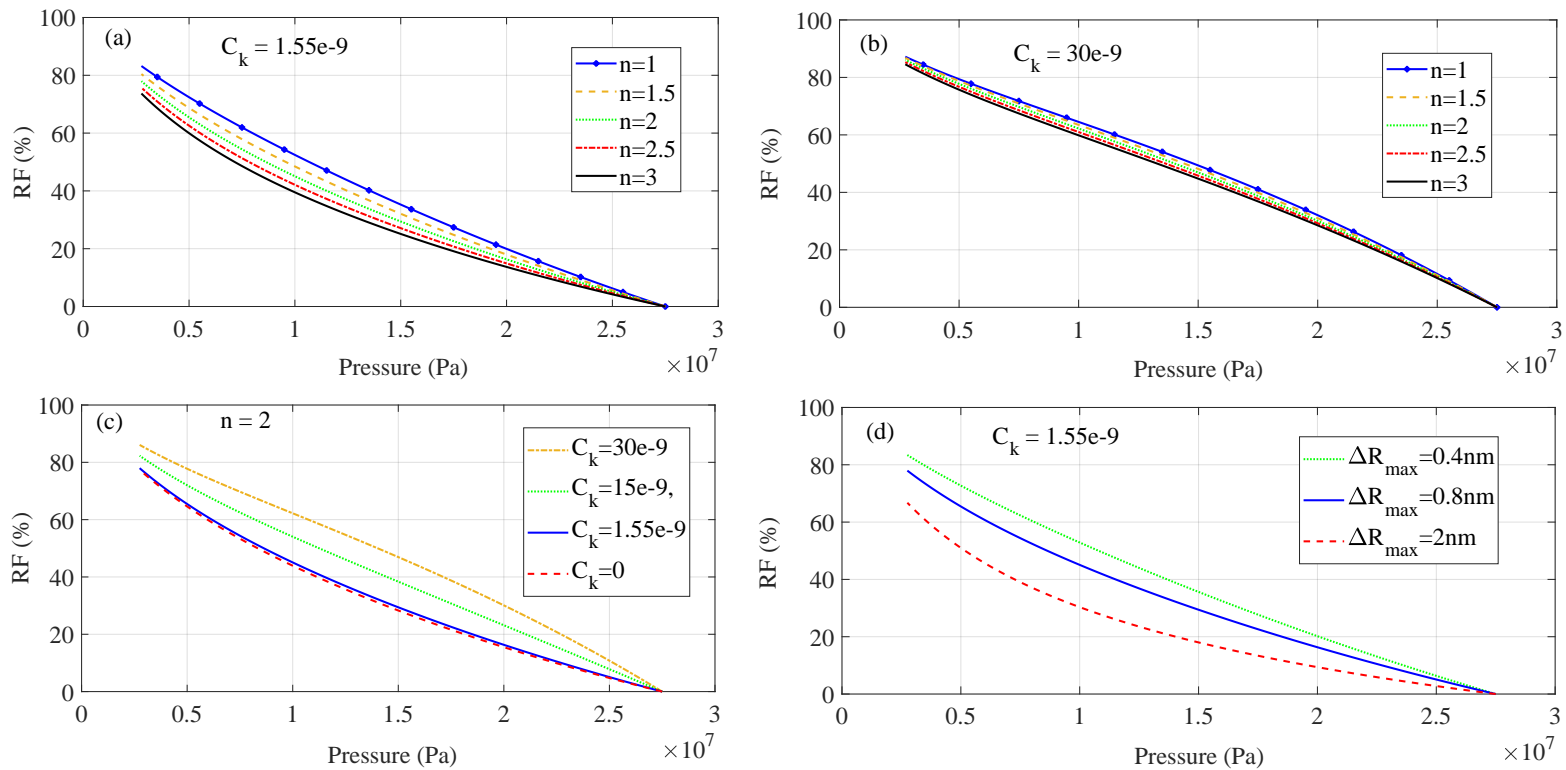

Fig. 8. Recovery factor $R F$ vs pressure for variations of geometry parameter $n$ for a low compressibility (a) and high compressibility (b), variation of compressibility (c) and $\Delta R_{\max }$ (d).

adsorbed fraction is low, the relation between recovery and pressure becomes more linear in accordance with Eqs. (59) and (57), see Fig. 8(a).

\subsubsection{Permeability}

Intrinsic permeability is plotted against pressure in Fig. 9 for the presented cases. As noted from Eq. (42) it is a function of effective radius, effective porosity (where tortuosity is included) and geometry, as described by the parameters $R_{e}$, $\phi_{e}, n$. Higher $n$ shifts more of the volumetric flow towards the pore noflow boundary, meaning the intrinsic permeability is reduced (Figs. 9(a) and 9(b)). High compressibility reduces the pore radius at low pressures which reduces permeability
(Fig. 9(c)). Desorption makes more of the pore space available for flow and increases permeability at low pressure, especially visible in less compressible cases (Fig. 9(d)).

The same plots are shown for apparent permeability in Fig. 10. Slip tends to increase permeability at low pressure and low effective radius which gives significant contributions in nanosize pores (Darabi et al., 2012). The apparent permeability is increasing with lowered pressure in all example cases, except in the high compressibility cases where reduction in intrinsic permeability is more dominating.

In supplementary material (SM) Fig. 1 apparent permeability is plotted against effective porosity for the considered pressures. In low compressibility cases (SM Figs. 1(a) and 

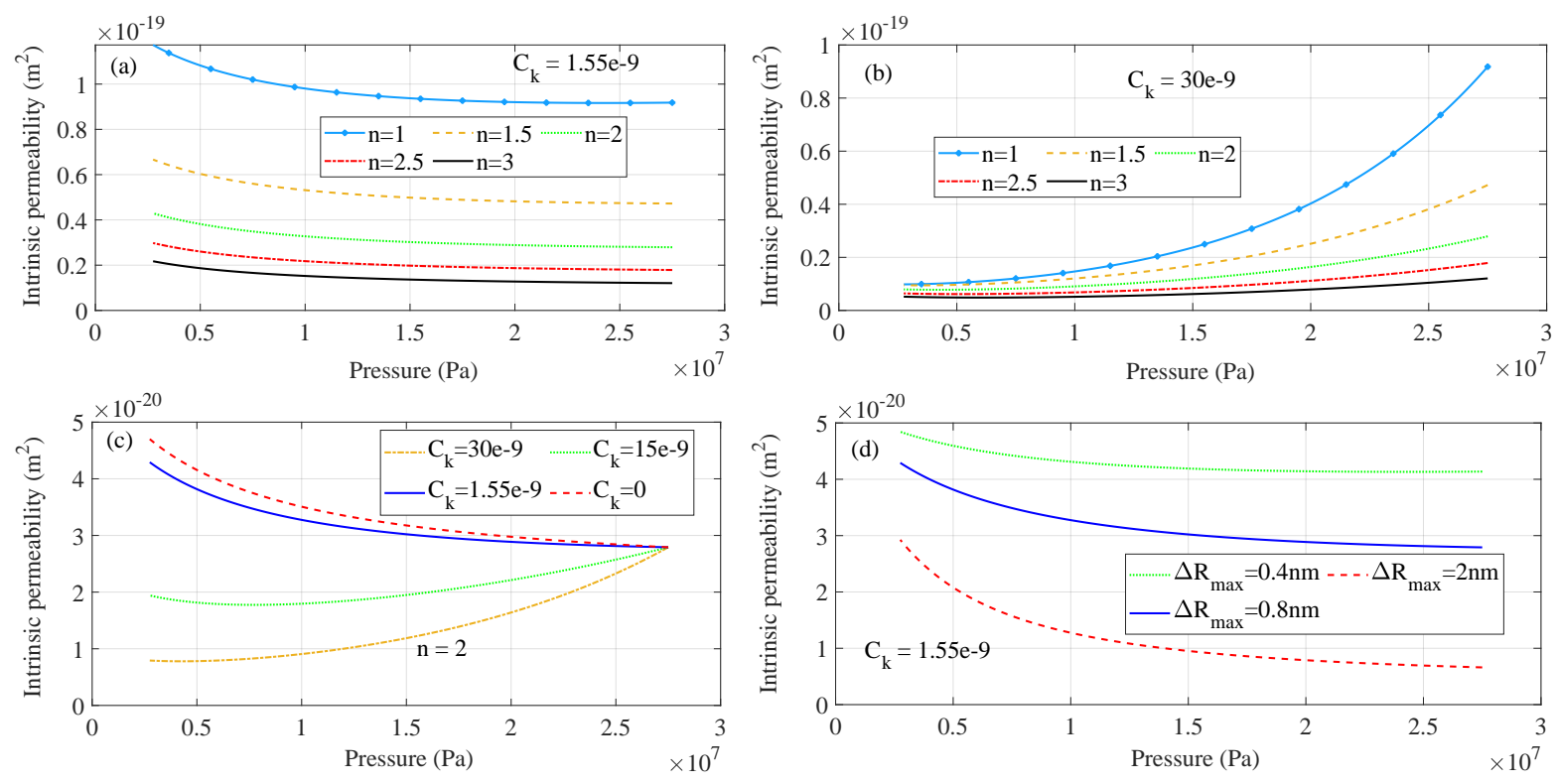

Fig. 9. Intrinsic permeability vs pressure for variations of geometry parameter $n$ for a low compressibility (a) and high compressibility (b), variation of compressibility (c) and $\Delta R_{\max }$ (d).
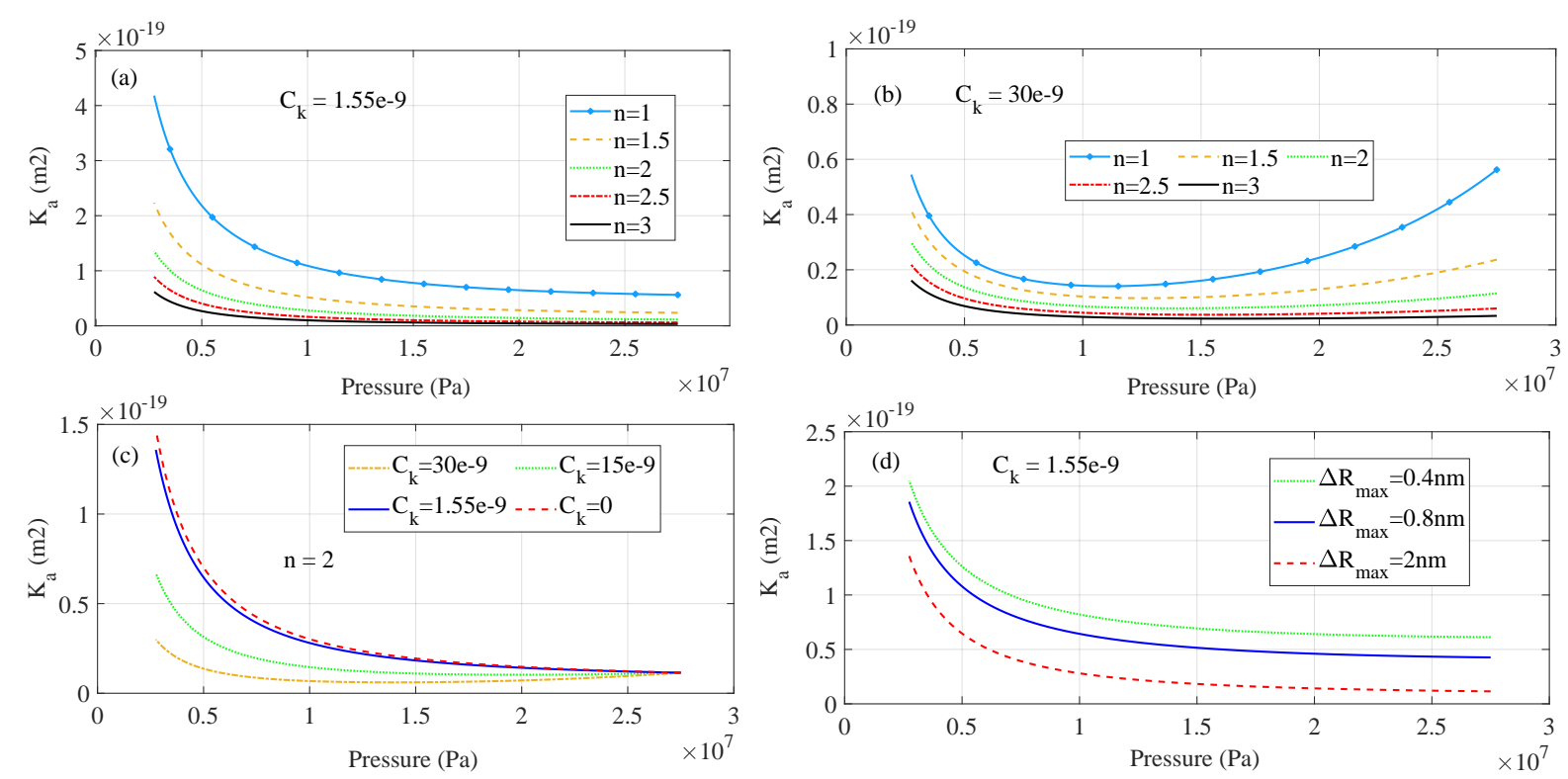

Fig. 10. Apparent permeability vs pressure for variations of geometry parameter $n$ for a low compressibility (a) and high compressibility (b), variation of compressibility (c) and $\Delta R_{\max }$ (d).

1(d)) the trend is monotonous and controlled by the increased effective radius $R_{e}$ from desorption at constant pore radius $R$. Lower apparent permeability then correlates with lower effective porosity. For compressible cases however (SM Figs. 1(b) and 1(c)), the effective porosity first reduces and then increases with lowered pressure. The apparent permeability also reduces at high pressure and increases at low pressures, but the mechanism is also related to slip which results in nonunique porosity-permeability relations.

\subsection{Dynamic simulation results}

In this section, the dynamic interplay of all properties discussed in the previous section will be examined using transient simulations. The reference values are applied including an initial pore radius of $4 \mathrm{~nm}$, initial porosity 0.06 and system length $10 \mathrm{~m}$.

\subsubsection{Introduction}

Fig. 11 shows the effect of compressibility on gas production by illustrating the evolution of recovery factor (a), average porosity (b), average free gas saturation (c) and average apparent permeability (d). These parameters are plotted against the square root of time for $30000 \mathrm{~d}$.

Higher compressibility reduces permeability (Fig. 11(d)) and delays recovery, but the end recovery is higher (Fig. 11(a)). Plotted against the square root of time, recovery is completely 

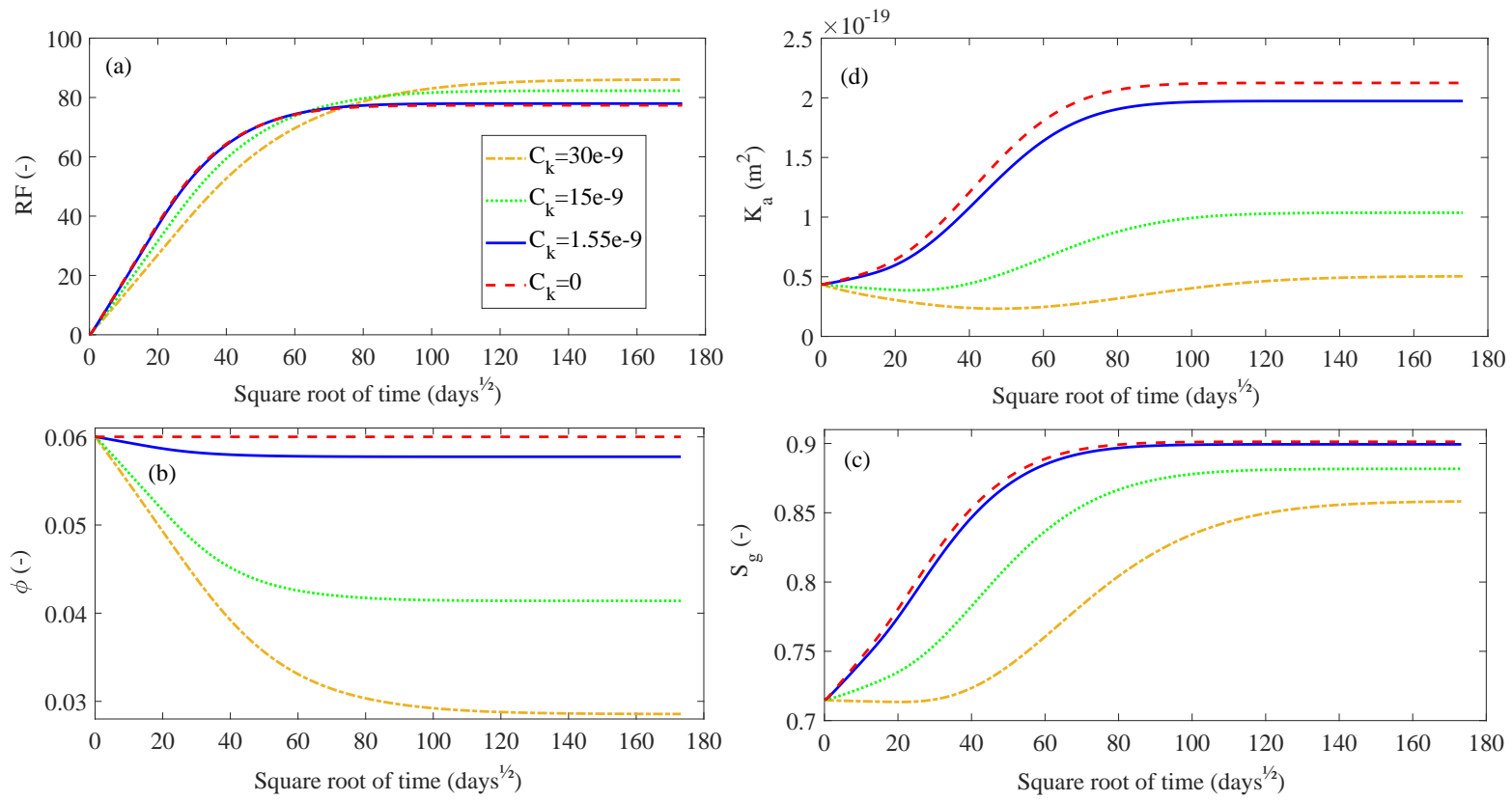

Fig. 11. Dynamic results from variation of compressibility $\left(c_{k}\right)$ : Recovery $R F$ (a), average porosity $\phi(b)$, average gas saturation $S_{g}(\mathrm{c})$ and average apparent permeability (d).
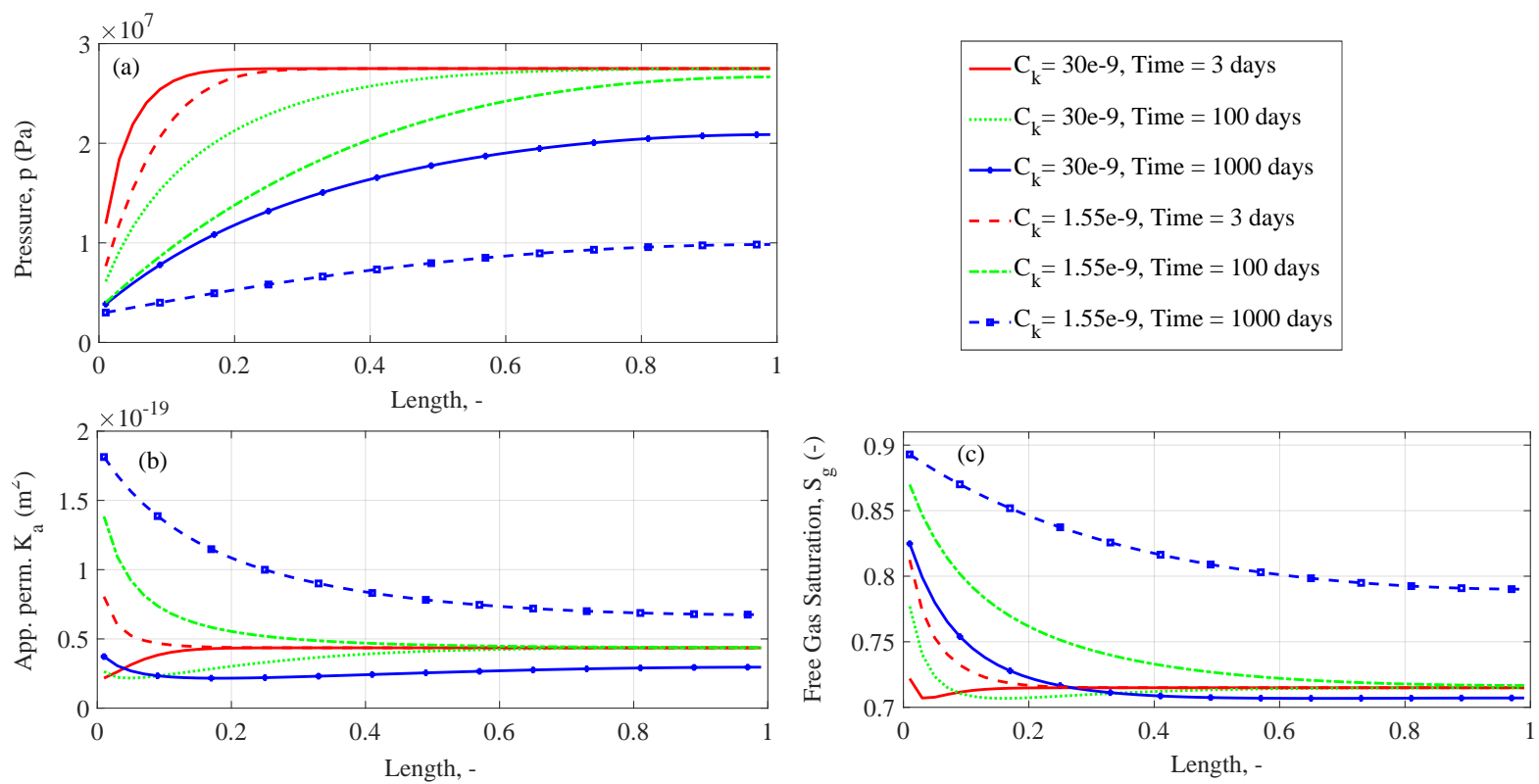

Fig. 12. Distributions of pressure $p$ (a), apparent permeability $K_{a}$ (b) and free gas saturation $S_{g}$ (c) for a high compressibility (solid lines) and the low base case compressibility (dashed lines).

linear at early time as predicted in Andersen (2021a). Porosity declines more with higher compressibility (Fig. 11(b)). The higher compressibility gives smaller pore radius, but same adsorption layer thickness and hence generally lower free gas saturation (Fig. 11(c)).

Spatial distributions are presented of pressure (Fig. 12(a)), apparent permeability (Fig. 12(b)) and free gas saturation (Fig. 12(c)) at different times for a high and low (base) compressibility. The pressure profile has a steep gradient initially which reduces at later times (Fig. 12(a)). The cases with high compressibility are delayed as the pore space deformation acts as a buffer. As the well indicates a fixed pressure at $x=0$, all the spatial profiles converge at $x=0$ to properties determined by the well pressure (a finer grid would show this even clearer). For low compressible cases, the highest apparent permeability is found at the well with values decreasing monotonously into the system (dashed lines in Fig. 12(b)). The apparent permeability is increased by slip and increased gas saturation (dashed lines in Fig. 12(c)) at low pressures near the well. For high compressibility cases the net effect of reduced pressure is a lower apparent permeability due to pore radius reduction, despite that gas saturation and slip increase. 

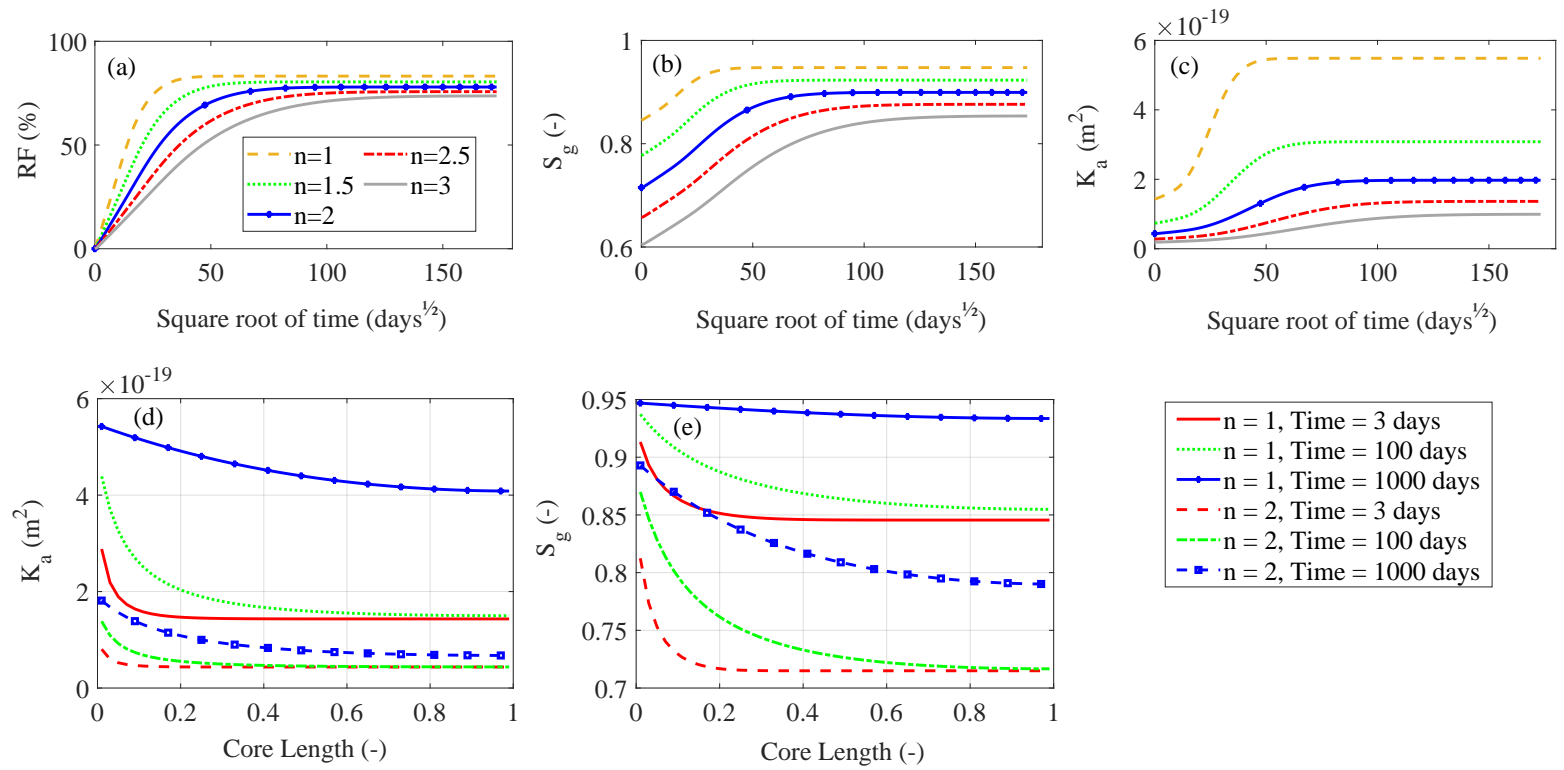

Fig. 13. Dynamic results from variation of geometry parameter $n$. Recovery $R F$ (a), average gas saturation $S_{g}$ (b) and average apparent permeability $K_{a}$ (c) plotted against square root of time. Spatial profiles are given of apparent permeability (d) and free gas saturation (e) for the cases $n=1$ (solid lines) and $n=2$ (dashed lines).

\subsubsection{The role of n-sphere geometry}

The effect of the geometrical parameter $n$ is investigated regarding the evolution of $R F$ (Fig. 13(a)), $S_{g}$ (b) and $K_{a}$ (c) over 30000 days of production. The base compressibility $1.55 \times 10^{-9} \mathrm{~Pa}^{-1}$ was used, resulting in minor changes of porosity. The pore radius is then close to constant and as noted in Fig. 5(a) the fraction $\Delta R / R$, and hence the effective radius $R_{e}$, reduces towards lower pressure with little dependence on $n$. On the other hand, there is more gas adsorbed per volume and thus lower free gas saturation at high $n$, see Fig. 13(c). The intrinsic permeability is considered proportional to $S_{g}$ in Eq. (42) through $\phi_{e}$ (note that $R_{e}$ stays the same at a given pressure) and high $n$ cases therefore have lower intrinsic permeability (Fig. 13(d)) and slower recovery (Fig. 13(a)) than low $n$ cases. Higher $n$ cases also reach lower end recovery as more of the gas is adsorbed and difficult to produce. Fig. 13 also illustrates distributions of apparent permeability (d) and gas saturation (e) for two cases where solid lines represent $n=1$ and dashed lines represent the base case $n=2$. Faster recovery in the low $n$ case can be linked to the higher free gas saturations and apparent permeabilities.

\subsubsection{Role of maximum adsorption layer thickness}

The effect of maximum adsorption layer thickness is investigated for three cases $\Delta R_{\max }=0.4,0.8$ and $2 \mathrm{~nm}$ corresponding to initial adsorbed gas fractions $0.27,0.47$ and 0.78 . Fig. 14 shows the results in terms of $R F$ (a), free gas saturation $S_{g}$ (b) and apparent permeability (c). The base compressibility yields negligible porosity changes. Both higher end recovery and recovery at a given time are achieved for low $\Delta R_{\max }$, i.e., when more of the shale gas is in free form (green curve). With low $\Delta R_{\max }$ the pores are more open for flow as $R_{e}$ and $S_{g}$ are larger, giving higher apparent permeability.
Spatial distributions are plotted for two cases $\Delta R_{\max }=0.8$ and $2 \mathrm{~nm}$. When $\Delta R_{\max }$ is low, the free gas saturation (d) is higher and so is the apparent permeability (e). Accordingly, recovery goes faster for low $\Delta R_{\max }$. Note that the change in free saturation is higher when $\Delta R_{\max }$ is high (from initially 0.38 to 0.75 vs from 0.72 to 0.9 ), The reason is that for low $\Delta R_{\max }$ the desorption occurs in the outermost layers with most volume and hence most impact on saturation.

\subsubsection{The importance of compressibility and adsorption layer}

Fig. 15 illustrates the evolution of recovery factor for four different cases based on different influence of compressibility and desorption. All cases have same initial conditions of pore radius, porosity and adsorbed layer thickness as given by the reference parameters. Two cases use a zero compressibility (red and orange curves), while the other two (blue and green curves) use a high compressibility of $30 \times 10^{-9} \mathrm{~Pa}^{-1}$. Further, two cases (green and orange curves) consider desorption of the adsorbed layer with pressure depletion (as before), while the other two (blue and red curves) assume the layer is not desorbed but remains at its initial thickness regardless of pressure depletion, i.e., $\Delta R=\Delta R\left(p_{0}\right)=0.8 \mathrm{~nm}$.

For these cases, the (reference) initial adsorbed mass fraction is $47 \%$. The lowest end recovery (orange curve) is obtained when gas does not desorb and the pores are not compressed. If the pores are compressed but gas does not desorb (green curve) the recovery is slowed down by permeability reduction, but end recovery is increased.

The fastest recovery is seen when gas desorbs and the pores are not compressed (red curve). Then permeability is increased by reduction in the adsorbed layer thickness and the pore radius is maintained. However, the highest end recovery is seen when accounting for both desorption and pore compression 

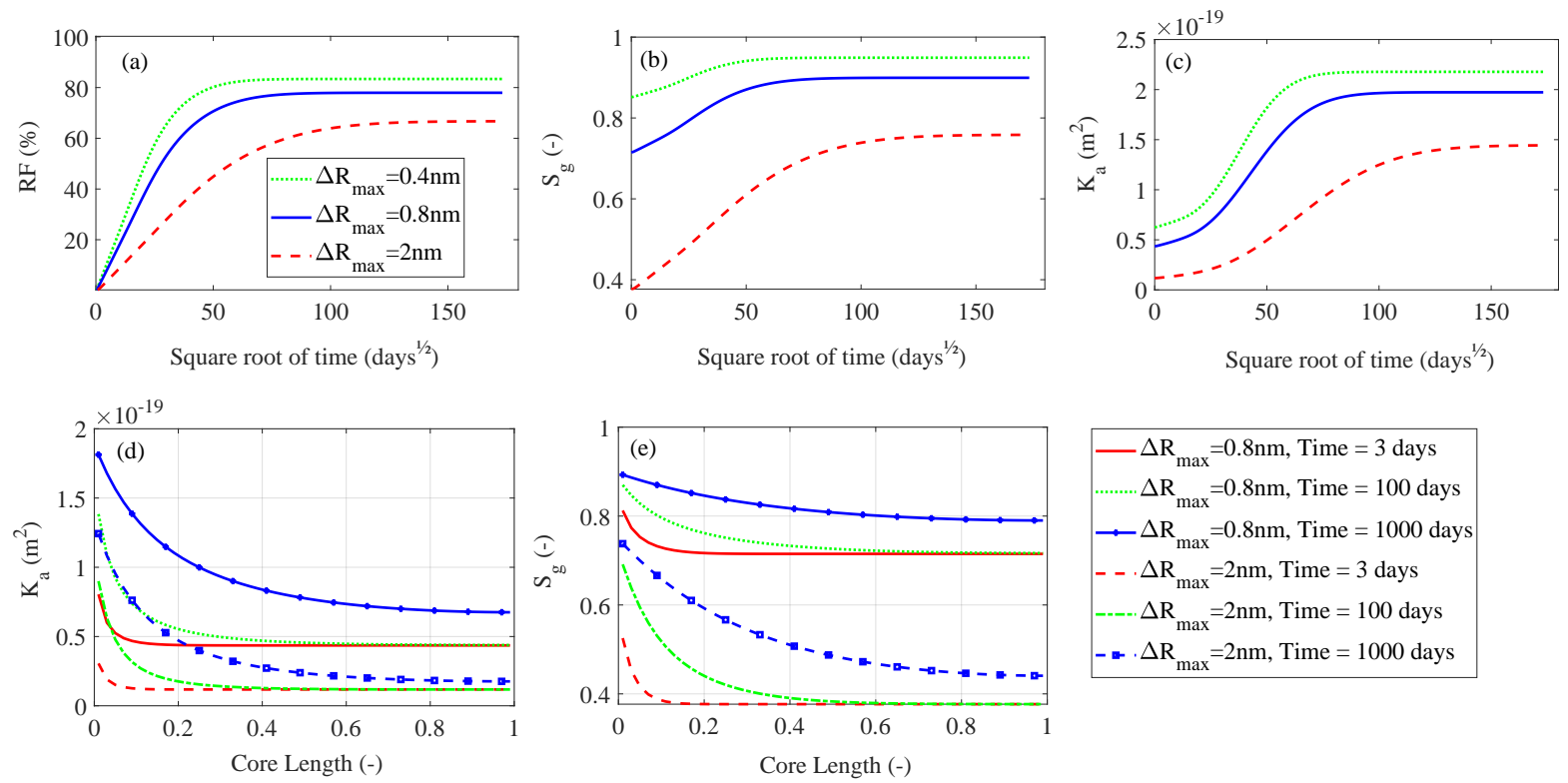

Fig. 14. Dynamic results from variation of maximum adsorption layer thickness $\Delta R_{\max }$ : Recovery $R F$ (a), average gas saturation $S_{g}$ (b) and average apparent permeability (e) are plotted against the square root of time. Spatial distributions are given of apparent permeability $K_{a}$ (d) and free gas saturation $S_{g}$ (e) for the cases $\Delta R_{\max }=0.8 \mathrm{~nm}$ (solid lines) and $\Delta R_{\max }=2 \mathrm{~nm}$ (dashed lines).

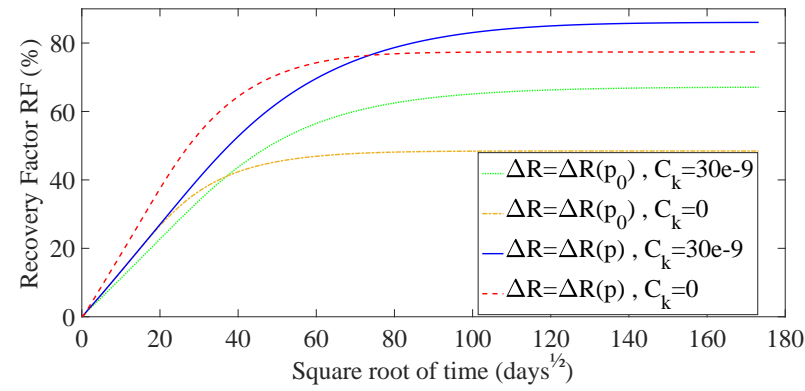

Fig. 15. Recovery factor $R F$ vs time for four cases: Zero compressibility with desorption (red) and without desorption (orange) and high compressibility of $30 \times 10^{-9} \mathrm{~Pa}^{-1}$ with desorption (blue) and without desorption (green).

(blue curve). Then adsorbed gas is produced and pore volume is compacted taking out more of the free gas. However, the reduction of pore radius reduces permeability and slows down recovery compared to the incompressible case.

\section{Conclusions}

A mathematical model has been derived for shale gas production considering the interplay of free and adsorbed gas in $n$-spherical pores where the adsorbed phase layer has a thickness of comparable dimension as the pore radius. A higher $n$ indicates more spherical pores, while a lower $n$ means more fracture shaped pores. The role of geometric and intrinsic properties were investigated under static and dynamic conditions and a consistent model was presented for variation between pore radius, adsorption layer thickness, porosity and apparent permeability. The permeability model yields the established expressions for a cylinder or fracture as special cases. The main findings are:

- For the same porosity and adsorbed layer thickness (same pressure), in more spherical shaped pores (higher $n$ ) a greater volume fraction of the pores is filled by the adsorbed gas since it is attached to the parts of the pores where a layer takes more volume.

- Pressure depletion causes a reduction in the adsorbed layer thickness, and if the shale is compressible, also the pore radius. The change in adsorbed layer thickness with pressure is low at high pressure and greater at low pressure. Pore radius changes more linearly with pressure. The free gas saturation can thus increase with pressure depletion (time) for low compressibility cases, or reach a minimum before increasing in high compressible cases.

- A larger fraction adsorbed gas results in reduced permeability by narrower pores, less linear relation between recovery and pressure and lower end recovery fraction. This is due to the low desorption taking place at high pressures compared to low pressures. Mainly free gas is produced at early times. Systems with higher adsorbed gas fraction see lower recovery at a given pressure.

- Apparent permeability increased monotonously towards the well where the pressure was lowest for incompressible cases. That is because desorption removed more of the adsorbed layer and because of higher slip factor with lower pressure. For compressible cases, the reduction in pore radius near the well could be more dominating and lead to a net reduction in permeability towards the well.

- Compressibility and desorption can both affect end recovery and recovery rate. Higher end recovery is seen when accounting for desorption and compressibility and vice versa. The fastest recovery is seen when accounting for desorption and not compressibility as the effective pore radius and permeability increases. 


\section{Acknowledgement}

Andersen acknowledges the Research Council of Norway and the industry partners, ConocoPhillips Skandinavia AS, Aker BP ASA, Vår Energi AS, Equinor ASA, Neptune Energy Norge AS, Lundin Norway AS, Halliburton AS, Schlumberger Norge AS, and Wintershall DEA, of The National IOR Centre of Norway for support.

\section{Conflict of interest}

The authors declare no competing interest.

Open Access This article is distributed under the terms and conditions of the Creative Commons Attribution (CC BY-NC-ND) license, which permits unrestricted use, distribution, and reproduction in any medium, provided the original work is properly cited.

\section{References}

Ambrose, R. J., Hartman, R. C., Diaz-Campos, M., et al. Shale gas-in-place calculations part I: New pore-scale considerations. SPE Journal, 2012, 17(1): 219-229.

Andersen, P. Ø. A semi-analytical solution for shale gas production from compressible matrix including scaling of gas recovery. Journal of Natural Gas Science and Engineering, 2021a, 95: 104227.

Andersen, P. Ø. Steady state gas flow from tight shale matrix subject to water blocking. SPE Journal, 2021b, (in Press).

Batchelor, C. K., Batchelor, G. K. An Introduction to Fluid Dynamics. Cambridge, UK, Cambridge University Press, 2000.

Berawala, D. S., Andersen, P. Ø. Evaluation of multicomponent adsorption kinetics for carbon dioxide enhanced gas recovery from tight shales. SPE Reservoir Evaluation \& Engineering, 2020a, 23(3): 1060-1076.

Berawala, D. S., Andersen, P. Ø. Numerical investigation of non-Darcy flow regime transitions in shale gas production. Journal of Petroleum Science and Engineering, 2020b, 190: 107114.

Berawala, D. S., Andersen, P. Ø., Ursin, J. R. Controlling parameters during continuum flow in shale-gas production: A fracture/matrix-modeling approach. SPE Journal, 2019, 24(3): 1-378.

Beskok, A., Karniadakis, G. E. Report: A model for flows in channels, pipes, and ducts at micro and nano scales. Microscale Thermophysical Engineering, 1999, 3(1): 4377.

Bird, G. A., Brady, J. M. Molecular Gas Dynamics and the Direct Simulation of Gas Flows (Vol. 5). Oxford, UK, Clarendon Press, 1994.

Bruggeman, V. D. Berechnung verschiedener physikalischer Konstanten von heterogenen Substanzen. I. Dielektrizitätskonstanten und Leitfähigkeiten der Mischkörper aus isotropen Substanzen. Annalen Der Physik, 1935, 416(7): 636-664.

Cao, P., Liu, J., Leong, Y. K. Combined impact of flow regimes and effective stress on the evolution of shale apparent permeability. Journal of Unconventional Oil and Gas Resources, 2016, 14: 32-43.

Carman, P. C. Flow of Gases Through Porous Media. New
York, USA, Academic Press, 1956.

Chen, F., Lu, S., Ding, X., et al. Evaluation of the density and thickness of adsorbed methane in differently sized pores contributed by various components in a shale gas reservoir: A case study of the Longmaxi Shale in Southeast Chongqing, China. Chemical Engineering Journal, 2019, 367: 123-138.

Civan, F. Reservoir Formation Damage-Fundamentals, Modeling, Assessment, and Mitigation. Gulf Professional Publishing, Amsterdam, the Netherlands, 2007.

Civan, F. Effective correlation of apparent gas permeability in tight porous media. Transport in Porous Media, 2010, 82(2): 375-384.

Civan, F., Rai, C. S., Sondergeld, C. H. Shale-gas permeability and diffusivity inferred by improved formulation of relevant retention and transport mechanisms. Transport in Porous Media, 2011, 86(3): 925-944.

Clarkson, C. R., Nobakht, M., Kaviani, D., et al. Production analysis of tight-gas and shale-gas reservoirs using the dynamic-slippage concept. SPE Journal, 2012, 17(1): 230-242.

Curtis, J. B. Fractured shale-gas systems. AAPG Bulletin, 2002, 86(11): 1921-1938.

Darabi, H., Ettehad, A., Javadpour, F., et al. Gas flow in ultratight shale strata. Journal of Fluid Mechanics, 2012, 710: 641-658.

Dong, J. J., Hsu, J. Y., Wu, W. J., et al. Stress-dependence of the permeability and porosity of sandstone and shale from TCDP Hole-A. International Journal of Rock Mechanics and Mining Sciences, 2010, 47(7): 1141-1157.

Evans, H. P. Volume of an n-dimensional sphere. The American Mathematical Monthly, 1947, 54(10): 592-594.

GeoQuest, S. EcLipse 100 (Black Oil): Reference Manual and Technical Description. Houston, 2014.

Gou, Q., Xu, S. Quantitative evaluation of free gas and adsorbed gas content of Wufeng-Longmaxi shales in the Jiaoshiba area, Sichuan Basin, China. Advances in GeoEnergy Research, 2019, 3(3): 258-267.

Higashi, K., Ito, H., Oishi, J. Surface diffusion phenomena in gaseous diffusion. I. Surface diffusion of pure gas. Nippon Genshiryoku Gakkaishi (Japan), 1963, 5: 4129274.

Hornyak, G. L., Dutta, J., Tibbals, H. F., et al. Introduction to Nanoscience and Nanotechnology. Boca Raton, USA, CRC Press, 2008.

Javadpour, F. Nanopores and apparent permeability of gas flow in mudrocks (shales and siltstone). Journal of Canadian Petroleum Technology, 2009, 48(8): 16-21.

Javadpour, F., Fisher, D., Unsworth, M. Nanoscale gas flow in shale gas sediments. Journal of Canadian Petroleum Technology, 2007, 46(10): 55-61.

Jiang, J., Yang, J. Coupled fluid flow and geomechanics modeling of stress-sensitive production behavior in fractured shale gas reservoirs. International Journal of Rock Mechanics and Mining Sciences, 2018, 101: 1-12.

Karniadakis, G., Beskok, A., Aluru, N. Microflows and Nanoflows: Fundamentals and Simulation. Berlin, Germany, Springer Science \& Business Media, 2006.

Klewiah, I., Berawala, D. S., Walker, H. C. A., et al. Review 
of experimental sorption studies of $\mathrm{CO}_{2}$ and $\mathrm{CH}_{4}$ in shales. Journal of Natural Gas Science and Engineering, 2020, 73: 103045.

Langmuir, I. The constitution and fundamental properties of solids and liquids. Part I. Solids. Journal of the American Chemical Society, 1916, 38(11): 2221-2295.

Lee, A. L., Starling, K. E., Dolan, J. P., et al. Viscosity correlation for light hydrocarbon systems. AIChE Journal, 1964, 10(5): 694-697.

Li, M., Tang, Y. B., Bernabé, Y., et al. Percolation connectivity, pore size, and gas apparent permeability: Network simulations and comparison to experimental data. Journal of Geophysical Research: Solid Earth, 2017, 122(7): 49184930.

Li, Z. Z., Min, T., Kang, Q., et al. Investigation of methane adsorption and its effect on gas transport in shale matrix through microscale and mesoscale simulations. International Journal of Heat and Mass Transfer, 2016, 98: 675686.

Loeb, L. B. The Kinetic Theory of Gases. North Chelmsford, USA, Courier Corporation, 2004.

Memon, A., Li, A., Jacqueline, N., et al. Study of gas sorption, stress effects and analysis of effective porosity and permeability for shale gas reservoirs. Journal of Petroleum Science and Engineering, 2020, 193: 107370.

Mengal, S. A., Wattenbarger, R. A. Accounting for adsorbed gas in shale gas reservoirs. Paper SPE 141085 Presented at SPE Middle East Oil and Gas Show and Conference, Manama, Bahrain, 25-28 September, 2011.

Montgomery, S. L., Jarvie, D. M., Bowker, K. A., et al. Mississippian Barnett Shale, Fort Worth basin, northcentral Texas: Gas-shale play with multi-trillion cubic foot potential. AAPG Bulletin, 2005, 89(2): 155-175.

Moridis, G. J., Blasingame, T. A., Freeman, C. M. Analysis of mechanisms of flow in fractured tight-gas and shalegas reservoirs. Paper SPE 139250 Presented at SPE Latin American and Caribbean Petroleum Engineering Conference, Lima, Peru, 1-3 December, 2010.

Pang, Y., Soliman, M. Y., Deng, H., et al. Analysis of effective porosity and effective permeability in shale-gas reservoirs with consideration of gas adsorption and stress effects. SPE Journal, 2017, 22(6): 1739-1759.

Patzek, T., Male, F., Marder, M. A simple model of gas production from hydrofractured horizontal wells in shales. AAPG Bulletin, 2014, 98(12): 2507-2529.

Peng, D. Y., Robinson, D. B. A new two-constant equation of state. Industrial and Engineering Chemistry Fundamentals, 1976, 15(1): 59-64.

Perez, F., Devegowda, D. Estimation of adsorbed-phase density of methane in realistic overmature kerogen models using molecular simulations for accurate gas in place calculations. Journal of Natural Gas Science and Engineering, 2017, 46: 865-872.

Ren, W., Li, G., Tian, S., et al. Adsorption and surface diffusion of supercritical methane in shale. Industrial and Engineering Chemistry Research, 2017, 56(12): 3446-
3455.

Shen, L., Chen, Z. Critical review of the impact of tortuosity on diffusion. Chemical Engineering Science, 2007, 62(14): 3748-3755.

Sheng, G., Javadpour, F., Su, Y. Effect of microscale compressibility on apparent porosity and permeability in shale gas reservoirs. International Journal of Heat and Mass Transfer, 2018, 120: 56-65.

Sheng, G., Su, Y., Zhao, H., et al. A unified apparent porosity/permeability model of organic porous media: Coupling complex pore structure and multi-migration mechanism. Advances in Geo-Energy Research, 2020, 4(2): 115-125.

Starling, K. E., Ellington, R. T. Viscosity correlations for nonpolar dense fluids. AIChE Journal, 1964, 10(1): 1115.

Strapoc, D., Mastalerz, M., Schimmelmann, A., et al. Geochemical constraints on the origin and volume of gas in the New Albany Shale (Devonian-Mississippian), eastern Illinois Basin. AAPG Bulletin, 2010, 94(11): 1713-1740.

Valkó, P. P., Lee, W. J. A better way to forecast production from unconventional gas wells. Paper SPE 134231 Presented at SPE Annual Technical Conference and Exhibition, Florence, Italy, 19-22 September, 2010.

Wang, J., Luo, H., Liu, H., et al. An integrative model to simulate gas transport and production coupled with gas adsorption, non-Darcy flow, surface diffusion, and stress dependence in organic-shale reservoirs. SPE Journal, 2017, 22(1): 244-264.

Wu, Y. S., Li, J., Ding, D., et al. A generalized framework model for the simulation of gas production in unconventional gas reservoirs. SPE Journal, 2014, 19(5): 845-857.

Xiong, X., Devegowda, D., Michel, G. G., et al. A fullycoupled free and adsorptive phase transport model for shale gas reservoirs including non-Darcy flow effects. Paper SPE 159758 Presented at SPE Annual Technical Conference and Exhibition, San Antonio, Texas, 8-10 October, 2012.

Yang, B., Kang, Y., You, L., et al. Measurement of the surface diffusion coefficient for adsorbed gas in the fine mesopores and micropores of shale organic matter. Fuel, 2016, 181: 793-804.

Yin, Y., Qu, Z. G., Zhang, J. F. An analytical model for shale gas transport in kerogen nanopores coupled with real gas effect and surface diffusion. Fuel, 2017, 210: 569-577.

Yu, W., Sepehrnoori, K. Simulation of gas desorption and geomechanics effects for unconventional gas reservoirs. Fuel, 2014, 116: 455-464.

Zarragoicoechea, G. J., Kuz, V. A. Critical shift of a confined fluid in a nanopore. Fluid Phase Equilibria, 2004, 220(1): 7-9.

Zhao, K., Du, P. Performance of horizontal wells in composite tight gas reservoirs considering stress sensitivity. Advances in Geo-Energy Research, 2019, 3(3): 287-303.

Zimmerman, R. W., Bodvarsson, G. S. Hydraulic conductivity of rock fractures. Transport in Porous Media, 1996, 23(1): 1-30. 


\section{Appendix A: Calculation of real gas density using the Peng-Robinson equation of state}

The Peng and Robinson (1976) equation of state is expressed below in terms of gas molar volume $V_{m}$ :

$$
\begin{gathered}
p=\frac{R_{g} T}{V_{m}-b}-\frac{a(T)}{V_{m}\left(V_{m}+b\right)+b\left(V_{m}-b\right)} \\
a=\frac{R_{g}^{2} T_{c}^{2}}{2.1870 p_{c}}\left[1+k\left(1-T_{r}^{0.5}\right)\right]^{2}, \quad b=\frac{R_{g} T_{c}}{12.8535 p_{c}}, \quad T_{r}=\frac{T}{T_{c}} \\
k=0.37464+1.5422 \omega-0.26992 \omega^{2}
\end{gathered}
$$

where $p_{c}$ and $T_{c}$ are critical pressure and critical temperature respectively, $T_{r}$ is reduced temperature and $\omega$ is the dimensionless acentric factor. Eq. (A-1) can also be expressed with the compressibility factor $z$ as a cubic equation:

$$
\begin{gathered}
z^{3}+c_{2} z^{2}+c_{1} z+c_{0}=0 \\
c_{2}=-1+B, \quad c_{1}=A-3 B^{2}-2 B, \quad c_{0}=-A B+B^{2}+B^{3} \\
A=\frac{p T_{c}^{2}}{2.1870 p_{c} T^{2}}\left[1+k\left(1-T_{r}^{0.5}\right)\right]^{2}, \quad B=\frac{p T_{c}}{12.8535 p_{c} T}
\end{gathered}
$$

By finding the roots of Eq. (A-4) the compressibility factor $z$ can be calculated.

\section{Appendix B: Calculation of gas viscosity}

The gas viscosity correlation by Lee et al. (1964) was applied, which was modified from Starling and Ellington (1964) to represent mixtures and pure components:

$$
\begin{gathered}
\mu=10^{-7} K \exp \left[X(T) \rho_{g}^{Y(T)}\right] \\
K=\frac{7.77+0.0063 M)\left(\frac{5}{9} T\right)^{1.5}}{122.4+12.9 M+\left(\frac{5}{9} T\right)} \\
X=2.57+\frac{1914.5}{\left(\frac{5}{9} T\right)}+0.0095 M, \quad Y=1.11+0.04 X
\end{gathered}
$$

where $T$ is absolute temperature in Kelvin and $M$ is molecular weight.

\section{Appendix C: Average properties}

Average values of porosity, gas saturation and gas pressure are given as:

$$
\bar{\phi}=\frac{1}{N_{x}} \sum_{i=1}^{N_{x}} \phi_{i}, \quad \bar{S}_{g}=\frac{\sum_{i=1}^{N_{x}} S_{g i} \phi_{i}}{\sum_{i=1}^{N_{x}} \phi_{i}}, \quad \bar{p}=\frac{\sum_{i=1}^{N_{x}} p_{i} \frac{S_{g i} \phi_{i}}{z_{i}}}{\sum_{i=1}^{N_{x}} \frac{S_{g i} \phi_{i}}{z_{i}}}
$$

The average 'mass' of free and adsorbed gas at a given time are:

$$
\bar{M}_{1}=\frac{1}{N_{x}} \sum_{i=1}^{N_{x}} \frac{p_{i} S_{g i}}{z_{i}} \phi_{i}, \quad \bar{M}_{2}=\frac{R_{g} T \rho_{a}}{M} \frac{1}{N_{x}} \sum_{i=1}^{N_{x}} \phi_{i}\left(1-S_{g i}\right)
$$




\section{Appendix D: Operator splitting and discretization}

The system in Section 2 is solved using an operator splitting approach (see Berawala and Andersen (2020a, 2020b) for examples of this approach in the shale gas context) by dividing the full system into more easily solved subsystems. The transport equation (Eq. (5)) is written with the notation:

$$
\partial_{t}\left\{\phi\left[\frac{p}{z} S_{g}+\hat{a}\left(1-S_{g}\right)\right]\right\}=\partial_{x}\left(D \partial_{x} p\right), \quad \hat{a}=\frac{R_{g} T \rho_{a}}{M}, \quad D(p)=\frac{p K_{a}}{z \mu}
$$

where $\hat{a}$ is a constant and $D$ is pressure and saturation dependent. This system is split into a 'Free gas transport' system where only free gas flow is considered and the adsorbed phase and porous medium are held undeformed; and a 'Redistribution' system where free gas, adsorbed gas and porosity are adjusted locally to meet equilibrium pressure constraints.

\section{D.1: Free gas transport}

For this system the spatial distributions of $S_{g}(x), \phi(x), D(x), z(x)$ are kept fixed with time during the splitting step $(t, t+\Delta T)$. The following equation then needs to be solved:

$$
\frac{S_{g}(x) \phi(x)}{z(x)} \partial_{t}(p)=\partial_{x}\left(D(x) \partial_{x} p\right)
$$

The matrix is divided into $N_{x}$ cells and an explicit discretization of Eq. (D-1) is used:

$$
\left(\frac{S_{g}}{z} \phi\right)_{i} \frac{p_{i}^{j+1}-p_{i}^{j}}{\Delta t}=\frac{\left(D \partial_{x} p\right)_{i+1 / 2}-\left(D \partial_{x} p\right)_{i-1 / 2}}{\Delta x}, \quad\left(i=1: N_{x}\right)
$$

where $i$ is the grid cell index and $j$ the time step. For central boundaries the fluxes are defined by:

$$
\left(D \partial_{x} p_{g}\right)_{i+1 / 2}=\frac{2}{\frac{1}{D_{i+1}}+\frac{1}{D_{i}}} \frac{p_{i+1}^{j}-p_{i}^{j}}{\Delta x}, \quad\left(D \partial_{x} p_{g}\right)_{i-1 / 2}=\frac{2}{\frac{1}{D_{i}}+\frac{1}{D_{i-1}}} \frac{p_{i}^{j}-p_{i-1}^{j}}{\Delta x}
$$

while the fluxes at the outer boundaries are given as:

$$
\left(D \partial_{x} p\right)_{1 / 2}=\frac{2}{\frac{1}{D_{1}}+\frac{1}{\infty}} \frac{p_{1}^{j}-p_{\text {well }}}{\frac{\Delta x}{2}}, \quad\left(D \partial_{x} p\right)_{N_{x}+1 / 2}=0
$$

The time step $\Delta t$ was selected sufficiently low to achieve stability of the solution.

\section{D.2: Redistribution}

While preserving the mass, gas is redistributed between free and adsorbed form and porosity is adjusted to the new pressure. No flow is assumed, giving:

$$
\partial_{t} M_{T}=0, \quad M_{T}=\phi\left[\frac{p S_{g}}{z}+\hat{a}\left(1-S_{g}\right)\right]
$$

where $M_{T}$ is the conserved property. Assume that the system was in equilibrium at an old state and that the transport solver was performed. Only the pressure in the first term (free gas) of $M_{T}$ will then have changed, while the remaining terms remain evaluated at the old state. The local value of $M_{T}$ is then:

$$
M_{T}=\phi\left(p_{\text {old }}\right)\left\{\frac{p_{\text {new }} S_{g}\left(p_{\text {old }}\right)}{z\left(p_{\text {old }}\right)}+\hat{a}\left[1-S_{g}\left(p_{\text {old }}\right)\right]\right\}
$$

After redistribution, all the terms should be evaluated at the same adjusted pressure $p_{a d j}$, while keeping the value of $M_{T}$ (mass conservation). This pressure is found by solving:

$$
f\left(p_{a d j}\right)=0
$$

where

$$
f=\phi\left(p_{a d j}\right)\left\{\frac{p_{a d j} S_{g}\left(p_{a d j}\right)}{z\left(p_{a d j}\right)}+\hat{a}\left[1-S_{g}\left(p_{a d j}\right)\right]\right\}-M_{T}
$$




\section{Appendix E: Convergence and numerical validation}

The reference case was simulated using different $\Delta T$ and different $N_{x}$. Resulting recovery against the square root of time is shown in SM Figs. 2(a) and 2(b) where these parameters were varied, respectively. Keeping $\Delta T$ sufficiently low leads the solution to converge. The late time recovery does not depend much on $\Delta T$, and only a minor impact can be noticed at early recovery for larger $\Delta T$. Sufficient convergence was considered achieved at $\Delta T=12 \mathrm{hrs}$ which was used as the reference value in our simulations. The model is also sufficiently converging for different $N_{x}$ and only minor effects are noticeable during the simulation period.

The code was also validated numerically by running a case under identical conditions in both our code and the commercial reservoir simulation software Eclipse 100 (Geoquest, 2014). A relatively simple case was implemented using ideal gas, pore compressibility $1.55 \times 10^{-9} \mathrm{~Pa}^{-1}$, viscosity $0.02 \mathrm{cP}$. Adsorption and slip effects were ignored. The initial porosity was 0.06 , and intrinsic permeability was set constant based on tortuosity $\tau=2.197$, pore radius $4 \mathrm{~nm}$ and the initial porosity, resulting in $54.5 \mathrm{nD}$. As indicated in SM Fig. 3 our code is able to give same results as the commercial model under comparable conditions. 